\title{
Equilibrium structure of a bidimensional asymmetric city
}

\author{
G. Carlier ${ }^{*}$ I. Ekeland ${ }^{\dagger}$
}

February 24, 2006

\begin{abstract}
We establish the existence of equilibrium configurations of a bidimensional, non-symmetric city with an arbitrary boundary and an arbitrary transportation cost. The (non-constant) densities of land used for business and for residence are equilibrium outcomes. The proof relies on the theory of optimal transportation
\end{abstract}

Keywords: spatial equilibrium, optimal transportation, Kantorovich duality.

We are particularly grateful to Robert E. Lucas Jr. and Esteban RossiHansberg for long and helpful discussions. We gratefully acknowledge the hospitality of the Department of Economics at the University of Chicago and the support of Pierre-André Chiappori. We thank Gilles Duranton, André Grimaud, Robert Helsley and Jacques-François Thisse for insightful comments.

${ }^{*}$ Université Paris 9 Dauphine, CEREMADE, carlier@ceremade.dauphine.fr.

${ }^{\dagger}$ Canada Research Chair in Mathematical Economics, Department of Mathematics, University of British Columbia, ivar.ekeland@math.ubc.ca. 


\section{Introduction}

This paper aims at explaining the internal structure of a city endogeneously, as an equilibrium solution between two different possible uses of land, for residence and for business. In our model, the driving force for concentration, and indeed for the existence of cities in the first place, is a positive externality of labor: the more workers are concentrated at a given place, the more productive each of them becomes. The driving force for dispersion, on the other hand, is the desire of every individual to spread his/her living quarters, occupying as much residential land as possible.

Firms will want to locate together, in order to benefit from the positive externality of labour. City dwellers will want to have their residence near their work, because of the cost of commuting. So firms will enter in competition with individuals for land use. It will be up to the landowners to apportion the land between business use and residential use, by renting the land to the highest bidder.

These ideas are classical in urban economics. They can be found in the books of Fujita [7] and of Fujita and Thisse [9]. Fujita and Ogawa, [15] and [10], were the first ones to combine them to into a mathematical model from which the internal structure of the city could be derived endogeneously. This path has then been followed by a number of authors, among which we want to single out Lucas and Rossi-Hansberg [14], which provided the inspiration for the present work.

Our model is of the open-city, absentee-landlord type. The indirect utility level within the city is fixed at some exogeneous level (presumably equal to the indirect utility level outside the city, so that there would be no incentive to migrate). The boundary of the city is given and the population level will adjust to achieve the prescribed level of utility in equilibrium. Firms produce a single good, which is used as numéraire. The land is owned by absentee landlords, who take away all the surplus of firms. In equilibrium, the market for land and the market for labour both clear.

Our model differs from the preceding ones in several respects. The lot size (amount of land occupied by one residence) is not assumed to be constant, as in [15], [9] or [1], but is determined endogeneously at each location by the equilibrium condition. The cost $c(x, y)$ of commuting from location $x$ to location $y$ is not assumed to be linear in the distance $|x-y|$; in fact, no specific functional form is assumed, all that is required is that $c$ is a continuous function of $x$ and $y$. Finally, and perhaps most importantly, our model is truly bidimensional: the (prescribed) shape of the city can be any bounded domain $\Omega$ in the plane. In the preceding literature, the city is assumed either to be "long and thin", in fact one-dimensional, or to have a 
circular structure, so that the given boundary is a circle and every location is fully characterized by its distance from the center. The recent model of Lucas and Rossi-Hansberg [14] is of that type; it is not clear, however, how their method could be extended to the non-circular case, and this question was at the origin of the present paper. To treat the general case, we had to change the cost structure (in [14], these are iceberg costs, time is lost in transportation, in our work commuting has a monetary cost as in [15] or [1]), and to develop a new mathematical method, which we hope is of general interest.

In the classical literature, the bid-rent function $\Psi(x, u)$ of households plays a crucial role: it is defined as the maximum rent that an individual is willing to pay at a location $x$ if he/she is to achieve utility level $u$ (see [7] and [9], particularly chapter 6). Here, we work directly on the revenue function $\varphi(x)$ and the wage function $\psi(y)$; here $\varphi(x)$ is the revenue that individuals living at location $x$ bring home (net of commuting costs), and $\psi(y)$ is the wage that firms located at $y$ pay people who work there. The functions $\varphi$ and $\psi$ are linked by free mobility of labor. Indeed, people living at $x$ rationally choose to work at a location $y$ as to maximize their revenue $\psi(y)-c(x, y)$, and the firms located at $y$ rationally choose to hire workers so as to minimize the wages. Hence, we have:

$$
\begin{aligned}
& \varphi(x)=\sup \{\psi(y)-c(x, y): y \in \Omega\} \\
& \psi(y)=\inf \{\varphi(x)+c(x, y): x \in \Omega\}
\end{aligned}
$$

We are not sure whether these relations have appeared earlier in the urban economics literature. They are crucial to our approach: if $\varphi(x)$ and $\psi(y)$ are known, everything else can be derived: the density of households, the density of firms, and the commuting patterns of workers. The mathematical tools to do so have been developed recently, within the framework of optimal transportation theory (see [20] for a recent survey), and will be used in our proofs.

Our main result is an existence theorem: we prove that there is an equilibrium structure of the city, for any prescribed utility level $\bar{u}$ and shape $\Omega$. We make very general (and, to us at least, very natural) assumptions on the utility function of consumers, the production function of firms, the externality of labour and the transportation costs. As a price to pay for this generality, people living at the same location $x$ do not all commute to the same location $y=s(x)$ for work: there is, for each location $x$, an equilibrium probability $P_{x}$ on $\Omega$, so that $P_{x}(y)$ is the probability of working at $y$, conditional on living at $x$. To have all individuals living at the same location $x$ commute to the same location $y=s(x)$ for work requires an additional assumption 
on the transportation cost, for instance that $c(x, y)=C(x-y)$, where $C$ is a smooth and strictly convex function. It will also be shown, under quite general assumptions, that, at equilibrium, some agents do have to commute: the autarky situation where every agent works where he/she lives cannot be an equilibrium.

Because of the presence of externalities, of transportation costs, and of various constraints, this is not an equilibrium result of Arrow-Debreu type. It shares, however, the defects of the Arrow-Debreu approach, namely that its very generality makes qualitative analysis difficult. We have no uniqueness result, and therefore no comparative statics. We have at the present time no efficient algorithm to compute the equilibrium structure, nor an example of interest simple enough to be solved analytically. All this can be traced back to the method of proof, which is by a fixed-point theorem in a suitable infinite-dimensional space.

It is not clear how to adapt our method to the closed-city model, where the total population, and not the utility level, is prescribed. On the other hand, we believe that our method can solve other problems, such as a multisectorial industry, inhomogeneous terrain, or residence externalities between households. We also hope that in the future we can develop a model where the shape of the city is not prescribed, but is another outcome of equilibrium.

The structure of the paper is as follows. The model is introduced in section 2. In section 3, we describe the rational behavior of agents, firms and landowners. In section 4 , we define equilibrium structures. In section 5, we state our main existence results under Cobb-Douglas specification of the production and utility functions and with transportation costs which are powers of the distance. In section 6 , we establish the existence of equilibria under more general assumptions. Section 7 is devoted to some specific comments and concluding remarks. Finally, proofs are gathered in the Appendix.

\section{The model}

The city consists of inhabitants, who work and consume, of firms and of land owners all of whom have to fit inside $\Omega$, a given open bounded and connected subset of $\mathbb{R}^{2}$. The city produces a single good which is both consumed and used as numéraire. We assume that whatever fraction of the production (respectively consumption) is not consumed (respectively produced) within the city is sold (respectively purchased from) abroad.

All the inhabitants are identical. They are equally productive and have the same utility function $(c, S) \mapsto U(c, S)$, where $c$ is their consumption of the produced good and $S$ is the amount of residential land they rent. We 
assume that $U$ is strictly concave, strictly increasing in both arguments and continuous. All workers are endowed with the same quantity of labor that they supply inelastically to the productive activity.

There is a commuting cost $c(x, y)$. Inhabitants who work at $y$ and live at $x$ get a wage $\psi$ and bring home $\psi-c(x, y)$.

All firms in the model have the same technology, with constant returns to scale. The production function per unit of land at a given location is $(z, n) \mapsto f(z, n)$, where $z$ is the productivity at this location and $n$ is number of workers per unit of business land. It is assumed that $f$ is continuous, strictly increasing with respect to both arguments and strictly concave with respect to $n$.

Given the overall density of jobs (number of jobs per unit of land, irrespective of its use) $\nu(x)$ at each location $x$ of the city, the productivity $z$ at $x$ reflects employment at neighboring locations through the formula:

$$
z(x)=Z_{\nu}(x):=g\left(\int_{\Omega} \rho(x, y) \nu(y) d y\right), x \in \Omega
$$

where the weighting kernel $\rho(.,$.$) is nonnegative and continuous on \bar{\Omega} \times \bar{\Omega}$ (hence uniformly continuous, $\bar{\Omega} \times \bar{\Omega}$ being compact) and $g$ is a continuous increasing bounded function $g: \mathbb{R}_{+} \rightarrow[\underline{z}, \bar{z}]$ with $+\infty>\bar{z}>\underline{z}>0$. The fact that $g$ is increasing reflects the positive externality of labour. The bounds on $g$ mean that productivity cannot tend to infinity even if the density of employment is very high (people get in each other's way), and that productivity is positive even if there is no employment around.

City land is owned by absentee landlords: agents who play no role in the production or consumption processes. It is assumed that they extract all surplus from workers and firms and that they let out their land to the highest bidder.

\section{Rational behavior}

We are looking for an equilibrium, that is, a situation where there is no incentive for inhabitants to change the place they live nor the place they work, and no incentive for firms to move. This definition will be made more precise later on. But already it has two important consequences: at equilibrium, all firms should make the same profit, and all inhabitants should have the same utility level, so that firms have no incentive to move their location and inhabitants no incentive to change their dwelling place. So, at equilibrium, all firms make profit 0 , the surplus being appropriated by the landlord, and 
all consumers have utility level $\bar{u}$, exogeneously given (for instance, it could be the reservation utility inhabitants could get from moving out of the city).

In this section, we first describe for given wages, revenues and productivities, the pointwise rational behaviour of consumer-workers, firms and landlords. In particular we shall determine rents for business use and for residential use as functions of wages, revenues and productivities. Then we deduce from free mobility of labor that optimal commuting choice imposes sharp restrictions on wages and revenues that take the form of conjugacy relations between these quantities.

\subsection{Agents}

Assume that inhabitants dwelling at $x \in \Omega$ have an available revenue (takehome pay, net of commuting costs) $\varphi$. It is is known that their utility level is $\bar{u}$. An inhabitant of the city dwelling at $x$ divides his revenue between consuming $c$ and renting land $S$ at respective prices 1 and $Q$. The available revenue $\varphi$ must be enough to yield utility $\bar{u}$. Defining:

$$
V(Q):=\min \{c+Q S: U(c, S) \geq \bar{u}\}
$$

we have $\varphi=V(Q)$ and since $V$ is obviously strictly increasing and continuous, one can invert this relation. The rent $Q$ is then given by $Q=Q(\varphi)=$ $V^{-1}(\varphi)$, and it is easy to see that $\varphi \mapsto Q(\varphi)$ is continuous and strictly increasing.

Inhabitants living at $x$ with revenue $\varphi$ then solve program (3.1) with $Q=Q(\varphi)$ which yields the optimal consumption level $c(\varphi)$ and residential space $S(\varphi)$. Let $N$ be the number of inhabitants housed at $x$. Since each one occupies $S(\varphi)$ units of land, we find that the relative density of residents (number of residents per unit of residential land) at $x$ only depends on the revenue $\varphi$ at $x$ and is given by:

$$
N=\frac{1}{S(\varphi)}=: N(\varphi)
$$

\section{$3.2 \quad$ Firms}

Assume that the wage paid at a location $y$ is $\psi$ and the productivity is $z$, then firms located at $y$ choose the level of employment at $y$ by solving the program:

$$
q(z, \psi):=\max \{f(z, n)-\psi \cdot n: n \geq 0\}
$$

Let $n(z, \psi)$ the corresponding optimal level of employment. The function $q(z, \psi)$ is the rent for business use and $n(z, \psi)$ is the relative density of jobs 
(number of jobs per unit of land used for production) at $y$ if productivity at $y$ is $z$ and wage is $\psi$.

\subsection{Landowners}

At location $x \in \Omega$, given rents for business and residential use $q(z(x), \psi(x))$ and $Q(\varphi(x))$, landowners determine a fraction $\theta(x) \in[0,1]$ devoted to business use that satisfies:

$$
\begin{aligned}
& q(z(x), \psi(x))>Q(\varphi(x)) \Rightarrow \theta(x)=1 \\
& q(z(x), \psi(x))<Q(\varphi(x)) \Rightarrow \theta(x)=0
\end{aligned}
$$

The previous conditions simply mean that the landowners rent their land to the highest bidders. If $q=Q$, landlords are indifferent between allocating land for residential or for business use, hence they rationally may choose any $\theta \in[0,1]$. The assumption that land is allocated to its highest value use clearly is a source of discontinuities that creates important mathematical difficulties.

\subsection{Free mobility of labor}

In what follows, we shall denote by $\operatorname{spt}(\mu)$ (respectively $\operatorname{spt}(\nu))$ the support of the density of residents (respectively of employment) $\mu$ (repectively $\nu$ ). We recall that the support of a nonnegative measure is the complement of the largest open set which is negligible for that measure. It is therefore natural to interpret $\operatorname{spt}(\mu)$ as the set of possible residential locations, a similar interpretation is also valid for $\operatorname{spt}(\nu)$. Let us denote by $\varphi(x)$ the available revenue at a possible residential location $x$ (i.e. $x \in \operatorname{spt}(\mu))$ and by $\psi(y)$ the wage paid at a possible job location $y$ (i.e $y \in \operatorname{spt}(\nu)$ ). The functions $\varphi$ and $\psi$ are linked by free mobility of labor. Indeed, people living at $x \in \operatorname{spt}(\mu)$ rationally choose to work at a location $y \in \operatorname{spt}(\nu)$ as to maximize their revenue $\psi(y)-c(x, y)$. Hence, we have:

$$
\text { for all } x \in \operatorname{spt}(\mu), \varphi(x)=\sup \{\psi(y)-c(x, y): y \in \operatorname{spt}(\nu)\} \text {. }
$$

This condition implies that if $y \in \operatorname{spt}(\nu)$ then:

$$
\psi(y) \leq \inf \{\varphi(x)+c(x, y): x \in \operatorname{spt}(\mu)\}
$$

but if the inequality was strict at $y$ then no one would work at $y$ : residents from every area would bring home more money by working somewhere else. 
Since $y \in \operatorname{spt}(\nu)$, there are firms located at $y$ and hence people who work there. Hence, we must have:

$$
\text { for all } y \in \operatorname{spt}(\nu), \psi(y)=\inf \{\varphi(x)+c(x, y): x \in \operatorname{spt}(\mu)\}
$$

This equality can also be interpreted as saying that firms located at $y$ try to attract residents who would work for the lowest wages.

In the sequel we shall say that a pair of functions $(\psi, \varphi)$ that satisfies (3.5) and (3.6) are conjugate to each other. Indeed the previous conjugacy relations are incentive-compatibility conditions which express free-mobility of labor. Note that the conjugacy relations written above not only depend on the cost function $c$ but also on $\mu$ and $\nu$ through the sets $\operatorname{spt}(\mu)$ and $\operatorname{spt}(\nu)$.

\subsection{Densities and transportation plans}

Given a productivity function $z$, a wage function $\psi$, a revenue function $\varphi$ and the landowner's choice function $\theta$ we get an overall density of residents $\mu$ and an overall density of jobs $\nu$ defined for all $(x, y) \in \Omega \times \Omega$ by:

$$
\mu(x)=(1-\theta(x)) N(\varphi(x)), \nu(y)=\theta(y) n(z(y), \psi(y))
$$

It will naturally be required at equilibrium that the total labor supply equals the total labor demand i.e. $\mu$ and $\nu$ have the same total mass.

A commuting plan (or transportation plan) is a nonnegative Borel measure $\gamma$ on $\Omega \times \Omega$ having $\mu$ as first marginal and $\nu$ as second marginal. In other words, $\gamma$ is a commuting plan if, for every Borel function $\varphi$ on $\Omega$, we have:

$$
\begin{aligned}
\int_{\Omega \times \Omega} \varphi(x) d \gamma(x, y) & =\int_{\Omega} \varphi(x) d \mu \\
\int_{\Omega \times \Omega} \varphi(y) d \gamma(x, y) & =\int_{\Omega} \varphi(y) d \nu
\end{aligned}
$$

We denote the set of commuting plans by $\Pi(\mu, \nu)$. The economic interpretation is as follows. For every $A$ and $B$ in $\Omega$, the number $\gamma(A \times B)$ is the mass of people living in $A$ and working in $B$. Associated with $\gamma$, there are two conditional probabilities $P_{x}^{\gamma}$ (probability of working at $y$ conditional on dwelling at $x$ ) and $P_{\gamma}^{y}$ (probability of dwelling at $x$ conditional on working at $y$ ), and we have:

$$
\gamma(A \times B)=\int_{A} P_{x}^{\gamma}(B) d \mu(x)=\int_{B} P_{\gamma}^{y}(A) d \nu(y) .
$$


which can alternatively be rewritten as:

$$
\gamma=\int_{\Omega} P_{x}^{\gamma} d \mu(x)=\int_{\Omega} P_{\gamma}^{y} d \nu(y)
$$

Free mobility of labor implies that at equilibrium, residents of $x$ choose a working place $y$ so as to maximize wage net of commuting cost, in view of (3.5)-(3.6), this can be formulated as:

$$
\psi(y)-\varphi(x)=c(x, y) \gamma \text {-a.e.. }
$$

Put differently, for $\mu$-a.e. $x$, for $P_{x}^{\gamma}$-a.e. $y$, one has

$$
\varphi(x)=\psi(y)-c(x, y)=\sup \left\{\psi\left(y^{\prime}\right)-c\left(x, y^{\prime}\right): y^{\prime} \in \operatorname{spt}(\nu)\right\} \text {. }
$$

\section{Equilibria}

Roughly speaking, an equilibrium in the present framework is a configuration (residents/jobs densities, wages/revenues, fraction of land devoted to business use, transportation plan and productivity) in which each agent, firm, landowner solves his pointwise maximization program, wages, revenues and the commuting plan are consistent with free-mobility of labor and finally, productivity reflects production externalities. This yields the formal definition:

Definition 4.1 An equilibrium for the transportation cost $c$ consists of a pair $(\mu, \nu) \in\left(L^{1}\left(\Omega, \mathbb{R}_{+}\right)\right)^{2}$, a continuous function $z$ on $\bar{\Omega}$, a pair $(\psi, \varphi) \in$ $\left(C^{0}\left(\Omega, \mathbb{R}_{++}\right)\right)^{2}$, a Borel measurable function $\theta$ with $\theta \in[0,1]$, and a nonnegative Borel measure $\gamma$ on $\Omega \times \Omega$ such that

1. $\int_{\Omega} \mu=\int_{\Omega} \nu>0$,

2. $z=Z_{\nu}$,

3. for all $(x, y) \in \Omega^{2}$ :

$$
\mu(x)=(1-\theta(x)) N(\varphi(x)), \nu(y)=\theta(y) n(z(y), \psi(y)),
$$

4. (3.3) and (3.4) hold,

5. $(\psi, \varphi)$ are conjugate in the sense that (3.5) and (3.6) hold, 
6. $\gamma \in \Pi(\mu, \nu)$ and:

$$
\psi(y)-\varphi(x)=c(x, y) \gamma-a . e . .
$$

Note that in the previous definition, the total population size is not fixed whereas the utility of residents $\bar{u}$ and the shape of the city are. As already mentioned, an important mathematical difficulty lies in the discontinuities created by the landowners' behaviour.

Of special interest are equilibria having the special feature that every consumer living at $x$ has the same job location $s(x)$. This means that the transportation plan $\gamma$ is in fact a transport map $s$. Mathematically speaking, the conditional probabilities are Dirac masses: $P_{x}^{\gamma}=\delta_{s(x)}$. Such equilibria will be called pure:

Definition 4.2 An equilibrium $(\mu, \nu, z, \psi, \varphi, \theta, \gamma)$ for the transportation cost $c$ is called pure if $\gamma$ is supported by the graph of some measurable transport map s $: \Omega \rightarrow \Omega$ which, by definition means that for all Borel subsets of $\Omega, A$ and $B$, one has:

$$
\gamma(A \times B)=\int_{A \cap s^{-1}(B)} \mu(x) d x
$$

Note that if the transportation map $s$ is as in the previous definition and since $\gamma \in \Pi(\mu, \nu)$, then $s$ is a measure preserving map between $\mu$ and $\nu$, i.e. for every Borel subset $B$ of $\Omega$, one has:

$$
\int_{s^{-1}(B)} \mu=\int_{B} \nu
$$

The existence of pure equilibria depends on the structure of the commuting cost and will be discussed in details in section 7.1.

\section{Existence of equilibria in the Cobb-Douglas case}

In this section, we prove the existence of equilibria under Cobb-Douglas specifications on the utility and production functions. In the next section, we will extend this existence result to more general situations. We single out the Cobb-Douglas case because it is transparent, and because the assumptions in section 6 are made on derived quantities, namely $n, N, q$ and $Q$, rather than on the fundamentals. 
So let us consider the Cobb-Douglas case:

$$
\begin{aligned}
f(z, n) & =z^{\gamma} n^{\alpha} \\
U(c, S) & =c^{\beta} S^{1-\beta}
\end{aligned}
$$

with $\gamma>0$ and $(\alpha, \beta) \in(0,1)^{2}$. Explicit computations lead to:

$$
\begin{aligned}
n(z, \psi) & =\left(\frac{\alpha z^{\gamma}}{\psi}\right)^{\frac{1}{1-\alpha}} \\
N(\varphi) & =\beta^{\beta /(1-\beta)} \bar{u}^{-1 /(1-\beta)} \varphi^{\beta /(1-\beta)} .
\end{aligned}
$$

And the rents for business and residential use are given by:

$$
\begin{aligned}
q(z, \psi) & =(1-\alpha) z^{\gamma /(1-\alpha)}\left(\frac{\alpha}{\psi}\right)^{\frac{\alpha}{1-\alpha}} \\
Q(\varphi) & =(1-\beta)\left(\frac{\beta^{\beta} \varphi}{\bar{u}}\right)^{1 /(1-\beta)}
\end{aligned}
$$

Let us further assume that $\Omega$ is an open bounded subset of $\mathbb{R}^{2}$ which is either convex (a polyhedron for instance) or has a smooth boundary. Finally, assume that the cost function $c$ is of the form:

$$
c(x, y)=M|x-y|^{\eta} \text { for all }(x, y) \in \mathbb{R}^{2} \times \mathbb{R}^{2} .
$$

with $M$ and $\eta$ positive constants. Note that we are in the strictly convex case when $\eta>1$ and in the linear case when $\eta=1$. Note also that when $\eta \in(0,1)$ (sublinear case), $c$ is not differentiable.

Theorem 5.1 Under the above assumptions, the following holds:

1. strictly convex case: if $\eta>1$ and $\alpha \geq 1 / 2$ then there exists at least one equilibrium for the cost $c$ and every equilibrium is pure,

2. sublinear case: if $0<\eta \leq 1$ and $\eta \geq 2(1-\alpha)$, then there exists at least one equilibrium for the cost $c$.

The proof is given in section 8. In fact, theorem 5.1 directly follows from the more general theorems 6.2 and 7.1 . 


\section{Existence of equilibria in the general case}

\subsection{Assumptions}

We shall prove existence of equilibria, under some assumptions listed below.

Assumption $1 \Omega$ is an open bounded and connected subset of $\mathbb{R}^{2}$ such that $|\partial \Omega|=0$, which satisfies an additional regularity condition: there exists $\delta_{0}>$ 0 and $\varepsilon_{0} \in(0, \pi)$ such that for every $y \in \Omega$, there is some $\varepsilon \in[0,2 \pi)$ such that $\Omega-y$ contains the intersection of the open ball of radius $\delta_{0}$ with a cone of angle $2 \varepsilon_{0}$ :

$$
\left\{y+(t \cos (v), t \sin (v)), t \in\left[0, \delta_{0}\right], v \in\left[\varepsilon-\varepsilon_{0}, \varepsilon+\varepsilon_{0}\right]\right\} \subset \bar{\Omega} .
$$

Note that the previous assumption is satisfied when $\Omega$ is a bounded domain of $\mathbb{R}^{2}$ which is either convex or of class $C^{1}$.

Let us define for $x \in \mathbb{R}^{2}$ :

$$
d(x, \Omega):=\inf \{|x-y|: y \in \Omega\},
$$

and for $r>0$, define:

$$
\bar{\Omega}_{r}:=\left\{x \in \mathbb{R}^{2}: d(x, \bar{\Omega}) \leq r\right\} .
$$

Assumption 2 There exists $r>0$ such that the cost function satisfies $c \in$ $C^{0}\left(\bar{\Omega}_{r} \times \bar{\Omega}_{r}, \mathbb{R}_{+}\right)$and $c(x, x)=0$ for all $x \in \bar{\Omega}_{r}$ (where $\bar{\Omega}_{r}$ is defined by (6.1)).

We define, for further reference, the modulus of continuity of $c$ with respect to its first argument by, for all $t \geq 0$ :

$$
\omega_{1}(t):=\sup \left\{\left|c\left(x_{1}, y\right)-c\left(x_{2}, y\right)\right|:\left(x_{1}, x_{2}, y\right) \in \bar{\Omega}_{r}^{3},\left|x_{1}-x_{2}\right| \leq t\right\} .
$$

Similarly, we define:

$$
\omega_{2}(t):=\sup \left\{\left|c\left(x, y_{1}\right)-c\left(x, y_{2}\right)\right|:\left(x, y_{1}, y_{2}\right) \in \bar{\Omega}_{r}^{3},\left|y_{1}-y_{2}\right| \leq t\right\} .
$$

Finally, set:

$\omega(t):=\sup \left\{\left|c\left(x_{1}, y_{1}\right)-c\left(x_{2}, y_{2}\right)\right|:\left(x_{1}, x_{2}, y_{1}, y_{2}\right) \in \bar{\Omega}_{r}^{4},\left|x_{1}-x_{2}\right|+\left|y_{1}-y_{2}\right| \leq t\right\}$.

Assumption 3 Both functions $N$ and $Q$ are continuous from $\mathbb{R}_{++}$to $\mathbb{R}_{++}$, increasing and

$$
\lim _{\varphi \rightarrow+\infty} N(\varphi)=\lim _{\varphi \rightarrow+\infty} Q(\varphi)=+\infty
$$


Assumption 4 Both functions $n$ and $q$ from $[\bar{z}, \underline{z}] \times \mathbb{R}_{++}$to $\mathbb{R}_{++}$are continuous, and satisfy the monotonicity relations:

$$
\left[z^{\prime} \leq z, \psi^{\prime}>\psi\right] \Longrightarrow n\left(z^{\prime}, \psi^{\prime}\right)<n(z, \psi) \text { and } q\left(z^{\prime}, \psi^{\prime}\right)<q(z, \psi) \text {. }
$$

Assumption 5 The function $h \mapsto n\left(\underline{z}, \omega_{2}(|h|)\right)$ is not integrable near $h=0$.

Note that assumptions 4 and 5 imply that for all fixed $z \in[\underline{z}, \bar{z}]$ :

$$
\lim _{\psi \rightarrow 0} n(z, \psi)=+\infty
$$

Assumption 6 The function $q$ satisfies:

$$
\lim _{\psi \rightarrow 0} q(\underline{z}, \psi)=+\infty .
$$

Before going further, let us note that conjugate functions satisfy the following regularity whose proof is elementary and therefore left to the reader.

Lemma 6.1 If $(\psi, \varphi)$ are conjugate in the sense that (8.2) and (8.3) hold, then for all $\left(x_{1}, x_{2}, y_{1}, y_{2}\right) \in \Omega^{4}$, one has:

$$
\left|\varphi\left(x_{1}\right)-\varphi\left(x_{2}\right)\right| \leq \omega_{1}\left(\left|x_{1}-x_{2}\right|\right),\left|\psi\left(y_{1}\right)-\psi\left(y_{2}\right)\right| \leq \omega_{2}\left(\left|y_{1}-y_{2}\right|\right) .
$$

Our existence proof relies heavily on Monge-Kantorovich optimal transportation theory. The basic results we shall use are recalled below, we refer to the recent book of Villani [20] for a detailed treatment of this rich theory and its wide spectrum of applications.

\subsection{Kantorovich duality}

Given two nonnegative Borel measures $\mu$ and $\nu$ on $\bar{\Omega}$ with common positive total mass, let us consider the Monge-Kantorovich optimal transportation problem:

$$
\left(\mathcal{M}_{\mu, \nu}\right) \inf \left\{\int_{\bar{\Omega} \times \bar{\Omega}} c(x, y) d \gamma(x, y): \gamma \in \Pi(\mu, \nu)\right\}
$$

If $\gamma \in \Pi(\mu, \nu)$ solves $\left(\mathcal{M}_{\mu, \nu}\right), \gamma$ is called an optimal transportation plan between $\mu$ and $\nu$ (for the cost $c$ ). It is customary in the Monge-Kantorovich theory to consider also the so-called dual problem:

$$
\left(\mathcal{D}_{\mu, \nu}\right) \sup _{\psi, \varphi}\left\{\int_{\bar{\Omega}} \psi d \nu-\int_{\bar{\Omega}} \varphi d \mu: \psi(y)-\varphi(x) \leq c(x, y),(x, y) \in \Omega^{2}\right\} .
$$

The main results from optimal transportation theory (we refer to [17] and [20] for proofs) that we shall need are summarized in the following: 
Theorem 6.1 Let $\mu$ and $\nu$ on $\bar{\Omega}$ be two Borel nonnegative measures on $\bar{\Omega}$ with common positive total mass and $c$ be a continuous transportation cost function on $\bar{\Omega} \times \bar{\Omega}$, then we have:

1. the supremum in $\left(\mathcal{D}_{\mu, \nu}\right)$ is attained by a pair $(\psi, \varphi)$ of functions that satisfy the conjugacy relations (8.2) and (8.3),

2. the infimum in $\left(\mathcal{D}_{\mu, \nu}\right)$ is attained by some $\gamma \in \Pi(\mu, \nu)$,

3. the duality relation $\inf \left(\mathcal{M}_{\mu, \nu}\right)=\sup \left(\mathcal{D}_{\mu, \nu}\right)$ holds,

4. if $(\psi, \varphi)$ solves $\left(\mathcal{D}_{\mu, \nu}\right)$ and $\gamma$ solves $\left(\mathcal{M}_{\mu, \nu}\right)$ then:

$$
\psi(y)-\varphi(x)=c(x, y) \gamma-a . e .
$$

5. if the pair $(\psi, \varphi)$ satisfies the conjugacy relations (8.2) and (8.3), and $\gamma \in \Pi(\mu, \nu)$ is such that:

$$
\psi(y)-\varphi(x)=c(x, y) \gamma \text {-a.e. }
$$

then $(\psi, \varphi)$ solves $\left(\mathcal{D}_{\mu, \nu}\right)$ and $\gamma$ solves $\left(\mathcal{M}_{\mu, \nu}\right)$.

We shall also use the following uniqueness result which is proven in section 8:

Proposition 6.1 If, in addition $c \in C^{1}(\bar{\Omega} \times \bar{\Omega}, \mathbb{R})$, and $(\mu, \nu)$ are absolutely continuous with respect to the Lebesgue measure and have positive densities on $\Omega$ then $\left(\mathcal{D}_{\mu, \nu}\right)$ admits a unique solution $(\psi, \varphi)$ up to equality almost everywhere and the addition of the same constant to both functions.

When the cost function is smooth (an assumption which is not realistic since it does not include the euclidean distance) Theorem 6.1 and proposition 6.1 are of particular interest in our equilibrium problem since they imply that wages and revenues can be deduced from densities. Indeed, those results from optimal transportation imply that, given a continuous positive density of residents $\mu$, and a continuous positive density of employment $\nu$, with same total mass there is a unique (up to the addition of a constant) pair of wage and revenue functions $\psi$ and $\varphi$ which induce a consistent transportation plan. We refer to section 8 for details and a rigorous construction. 


\subsection{Main result}

Our main existence result finally reads as:

Theorem 6.2 Under assumptions 1, 2, 3, 4, 5, 6, there exists at least one equilibrium for the transportation cost $c$.

To the best of our knowledge, theorem 6.2 and the general approach of the present paper, exhibit some features that seem to be novel. Firstly, our result is really two-dimensional and does not require any radial symmetry assumption as in [14] for instance. Precisely because we deal with a really twodimensional case, standard methods based on ordinary differential equations just don't work here. For special (quadratic) cost functions however, the existence of equilibria is, at least formally, linked to some fully nonlinear partial differential equations of Monge-Ampère type. Secondly, the cost functions we consider are quite general: they do not need to be smooth, to depend only on distance, or to have particular symmetry or convexity/concavity properties (however the convex case will be discussed in subsection 7.1). Finally, using the optimal transportation framework also allows to deduce commuting plans in an easy and general way from the wages and revenues patterns. Some generalizations are discussed in paragraph 7.3.

Most of the Appendix is devoted to the proof of theorem 6.2. The proof is quite long, and we wish at this point to briefly indicate its main steps:

- regularize the cost function and approximate the landowner's discontinuous behaviour by a continuous one, this leads to a notion of approximated equilibria we call $\varepsilon$-equilibria (see subsection 8.1),

- prove that $\varepsilon$-equilibria are associated to fixed-points of a certain operator defined on densities and related to the optimal transportation problem,

- prove that this operator satisfies the assumptions of Schauder's theorem so that there exist approximated equilibria,

- let the approximation parameter $\varepsilon$ tend to zero and prove that one can recover an equilibrium as a limit point of approximated equilibria.

\section{Concluding remarks}

\subsection{On cost functions and pure equilibria}

At this point, we aim to emphasize some important effect of the structure of commuting costs on equilibria. Indeed, when the cost satisfies a certain 
structural assumption that we call the generalized Spence-Mirrlees condition, at equilibrium commuting plans are really commuting maps. In other words, in such a case, every equilibrium is pure hence the commuting plan $\gamma$ is of the form $\gamma=\int_{\Omega} \delta_{s(x)} d \mu(x)$. Assuming that the cost function $c$ is smooth, the Spence-Mirrlees condition reads as the injectivity requirements:

$$
\begin{aligned}
& \nabla_{x} c\left(x, y_{1}\right)=\nabla_{x} c\left(x, y_{2}\right)=0 \Rightarrow y_{1}=y_{2}, \\
& \nabla_{y} c\left(x_{1}, y\right)=\nabla_{y} c\left(x_{2}, y\right)=0 \Rightarrow x_{1}=x_{2} .
\end{aligned}
$$

Assume now that $(\mu, \nu, z, \psi, \varphi, \theta, \gamma)$ is an equilibrium and write:

$$
\gamma=\int_{\Omega} P_{x}^{\gamma} d \mu(x)
$$

We know then (see formula (8.30)) that if $\varphi$ is differentiable at $x$ (which is the case a.e. by Rademacher's theorem and lemma 6.1) then, for $P_{x}^{\gamma}$ a.e. $y$ one has $\nabla \varphi(x)=-\nabla_{x} c(x, y)$. With (7.1), this in turn implies that the support of $P_{x}^{\gamma}$ is reduced to a single point $s(x)$ satisfying:

$$
\nabla \varphi(x)=-\nabla_{x} c(x, s(x))
$$

Under the Spence-Mirrlees assumption, at equilibrium, the job's location of an agent is then a deterministic function of his/her address. More is true: commuting is reversible in the sense that there is a reverse map $t\left(t=s^{-1}\right.$ in some weak sense) mapping job's location into the worker's adress. Combining the previous arguments with theorem 6.2 , we have the following result on existence of pure equilibria:

Theorem 7.1 If, in addition to assumptions 1, 2, 3, 4, 5 and 6, the cost function $c$ is smooth on $\bar{\Omega}_{r} \times \bar{\Omega}_{r}$ and satisfies (7.1)-(7.2) on $\bar{\Omega}_{r} \times \bar{\Omega}_{r}$ then there exists at least one equilibrium for the transportation cost $c$ and every equilibrium is pure.

It is well-known in optimal transportation theory that the Spence-Mirrlees assumption is important if one wants to find optimal maps rather than optimal plans (see [12], [4]). An important class of costs satisfying (7.1) (7.2) is the class of convex costs: $c(x, y)=C(x-y)$ with $C$ strictly convex, smooth and minimal at 0 . For this class of costs, considered by McCann and Gangbo [3], using the identity (7.3), we find that the commuting map $s$ is linked to the gradient of the revenue function by:

$$
s(x)=x-(\nabla C)^{-1}(-\nabla \varphi(x)) .
$$


In that case, the equilibrium condition of conservation of the densities leads to a certain fully nonlinear partial differential equation (of Monge-Ampère type if $\left.C(x)=|x|^{2}\right)$.

In the concave case $(c(x, y)=C(|x-y|)$ with $C$ strictly concave), the picture is totally different. In that case, also considered in [3], condition (7.1)(7.2) is satisfied on $\operatorname{spt}(\mu) \times \operatorname{spt}(\nu)$ only if those supports are disjoint, which is of course an irrelevant situation in our equilibrium setting. In that concave case, Mc Cann and Gangbo [3] actually characterized optimal transportation plans between $\mu$ and $\nu$ and proved that it involves some "splitting". The "mass" that is common to $\mu$ and $\nu$ stays where it is and after substracting this mass one is left with the optimal transportation of $(\mu-\nu)_{+}$to $(\nu-\mu)_{+}$. Put differently, at a given location $x$ where $\mu(x)>\nu(x)$, there are both residents working at $x$ and residents working at some location $s(x) \neq x$ with $\nu(s(x))>\mu(s(x))$.

\subsection{Equilibrium involves commuting}

Under quite natural additional assumptions, in our model, the autarky situation (in which every agent lives where he/she works, hence $\mu=\nu$ ) cannot happen at equilibrium. In other words, agents do have to commute at equilibrium. This in fact follows from production externalities and is quite independent from the rest of the paper. In addition to assumptions 1, 2, 3, 4, 5, 6 of the paper, let us further assume:

- the kernel $\rho$ is of the form $\rho(x, y)=f(|x-y|)$ with $f$ smooth and such that $f^{\prime}<0$ on $\mathbb{R}_{+}$,

- for all $\psi \in \mathbb{R}_{++}^{2} z \mapsto q(z, \psi)$ is increasing on $[\underline{z}, \bar{z}]$,

- the cost function $c$ is of class $C^{1}$ on $\bar{\Omega}^{2}$ and such that $c(x, y)>0$ when $(x, y) \in \bar{\Omega}^{2}$ and $x \neq y$.

Let us remark that since $c$ vanishes on the diagonal of $\bar{\Omega}^{2}$, the last condition implies $\nabla_{x} c(x, x)=\nabla_{y} c(x, x)=0$ for all $x \in \bar{\Omega}$. Under these assumptions, there necessarily is commuting at equilibrium, that is:

Proposition 7.1 Under assumptions 1, 2, 3, 4, 5, 6 and the additional assumptions of this paragraph, if $(\mu, \nu, z, \psi, \varphi, \theta, \gamma)$ is an equilibrium then $\mu \neq \nu$. 


\subsection{On zoning restrictions, space homogeneity and ex- ternalities}

In the present paper, we have considered the classical case of absentee landlords allocating land to its highest value use. Hence we have imposed conditions (3.3) and (3.4) on $\theta, z, \psi$, and $\varphi$ in the definition of equilibria. As already mentioned, because of discontinuities and zero densities, those conditions actually make the problem mathematically complicated. However, one can rule out zero densities, by assuming that there are zoning restrictions (imposed, say, by city regulations) that prevent the landlords from renting all their land either for one use only. Such regulations may be justified by urban planning and legal considerations; indeed, zoning restrictions, imposing a mixed use of land are used in many cities, Paris for instance. Our main result and constructions remain valid if one imposes such zoning restrictions, and the proofs are actually simpler. In fact, zoning restrictions are one of the mathematical artefacts that we use in section 8.1 to construct approximate equilibria. It should then be clear to the reader from our proof that theorem 6.2 still holds in models with additional zoning restrictions.

In our model, we have assumed that space in the city is homogenous from both consumers' and producers' point of view and we have taken production externalities given by formula (2.1). Even though such assumptions are classical, we claim that our existence proof and our optimal transportation approach can easily be adapted to the following cases:

- non homogenous space : location-dependent utility and production functions (due to purely geographical factors for instance),

- more general production externalities i.e. a productivity function of the form:

$$
z(x)=g\left(\int_{\Omega} \rho(x, y) \nu(y) d y, x\right)
$$

with a general function $g: g$ non monotonic with respect to its first argument (congestion effect, say), $g$ only depending on $x$ (exogenous productivity),....

- externalities (positive or negative depending on the phenomeon one aims to capture: attraction or repulsion) among residents : utility function at location $x$ depending on a parameter of the form:

$$
\int_{\Omega} \rho(x, z) \mu(z) d z
$$




\section{Appendix: proofs}

\section{$8.1 \quad \varepsilon$-equilibria}

One mathematical difficulty to prove existence of equilibria lies in the fact that one has to deal with measures that vanish on unknown sets and have discontinuities. This is due to conditions (3.3), (3.4) i.e. to the requirement that landowners allocate land to the highest bidder. One possibility to overcome this difficulty is to regularize the landowner's behavior as follows. Let $\varepsilon \in(0,1 / 2)$ and consider the program

$$
\max \left\{\theta q(z, \psi)+(1-\theta) Q(\varphi)-\frac{\varepsilon}{2} \theta^{2}: \varepsilon \leq \theta \leq 1-\varepsilon\right\}
$$

This determines the function $(z, \psi, \varphi) \mapsto \theta_{\varepsilon}(z, \psi, \varphi)$ by

$$
\theta_{\varepsilon}(z, \psi, \varphi)= \begin{cases}\varepsilon^{-1}(q(z, \psi)-Q(\varphi)) & \text { if } q(z, \psi)-Q(\varphi) \in\left[\varepsilon^{2}, \varepsilon-\varepsilon^{2}\right] \\ \varepsilon & \text { if } q(z, \psi)-Q(\varphi) \leq \varepsilon^{2} \\ 1-\varepsilon & \text { if } q(z, \psi)-Q(\varphi) \geq \varepsilon-\varepsilon^{2} .\end{cases}
$$

Finally define the functions:

$$
\begin{aligned}
& \widetilde{\mu}_{\varepsilon}(z, \psi, \varphi):=\left(1-\theta_{\varepsilon}(z, \psi, \varphi)\right) N(\varphi) \\
& \widetilde{\nu}_{\varepsilon}(z, \psi, \varphi):=\theta_{\varepsilon}(z, \psi, \varphi) n(z, \psi)
\end{aligned}
$$

And let us consider the free-mobility of labor conditions when there are jobs and residents all over the city:

$$
\begin{aligned}
& \varphi(x)=\sup \{\psi(y)-c(x, y): y \in \Omega\} \\
& \psi(y)=\inf \{\varphi(x)+c(x, y): x \in \Omega\}
\end{aligned}
$$

Definition 8.1 Let $\varepsilon \in(0,1 / 2)$. An $\varepsilon$-equilibrium for the transportation cost $c$ consists of a pair $(\mu, \nu) \in\left(L^{1}\left(\Omega, \mathbb{R}_{+}\right)\right)^{2}$, a continuous function $z$ on $\bar{\Omega}$, a pair $(\psi, \varphi) \in\left(C^{0}\left(\Omega, \mathbb{R}_{++}\right)\right)^{2}$ and a nonnegative Borel measure $\gamma$ on $\Omega \times \Omega$ such that

1. $\int_{\Omega} \mu=\int_{\Omega} \nu>0$,

2. $z=Z_{\nu}$,

3. for all $(x, y) \in \Omega^{2}$ :

$$
\mu(x)=\widetilde{\mu}_{\varepsilon}(z(x), \psi(x), \varphi(x)), \nu(y)=\widetilde{\nu}_{\varepsilon}(z(y), \psi(y), \varphi(y)),
$$


4. $(\psi, \varphi)$ are conjugate in the sense that (8.2) and (8.3) hold,

5. $\gamma \in \Pi(\mu, \nu)$ and:

$$
\psi(y)-\varphi(x)=c(x, y) \gamma-a . e . .
$$

\subsection{Existence of $\varepsilon$-equilibria}

In order to be able to deduce wages and revenues from densities in a unique way as in proposition 6.1, we first need to regularize the cost function as follows. Let $n$ be an integer, first set $\widetilde{c}_{n}(x, y):=\max (0, c(x, y)-\omega(2 / n))$ for all $(x, y) \in \bar{\Omega}_{r}^{2}$. For $n \geq r^{-1}$, define then for all $(x, y) \in \bar{\Omega}^{2}$ :

$$
c_{n}:=\left(\eta_{n} \star \widetilde{c}_{n}\right)(x, y)=\int_{B(0,1 / n)} \int_{B(0,1 / n)} \eta_{n}(u, v) \widetilde{c}_{n}(x-u, y-v) d u d v
$$

where $\eta_{n}(u, v):=n^{4} \eta(n u) \eta(n v)$ and $\eta$ is a $C^{\infty}$ nonnegative function with support in the unit ball of $\mathbb{R}^{2}$ and integral 1.

We let the reader verify that by construction, for every $n \geq r^{-1}$, we have the following:

- $c_{n} \geq 0$ on $\bar{\Omega}^{2}$ and $c_{n}(x, x)=0$ for all $x \in \bar{\Omega}$,

- for all $\left(x_{1}, x_{2}, y\right) \in \bar{\Omega}^{3},\left|c_{n}\left(x_{1}, y\right)-c_{n}\left(x_{2}, y\right)\right| \leq \omega_{1}\left(\left|x_{1}-x_{2}\right|\right)$,

- for all $\left(y_{1}, y_{2}, x\right) \in \bar{\Omega}^{3},\left|c_{n}\left(x, y_{1}\right)-c_{n}\left(x, y_{2}\right)\right| \leq \omega_{2}\left(\left|y_{1}-y_{2}\right|\right)$,

- $c_{n}$ converges uniformly to $c$ on $\bar{\Omega}^{2}$.

Also note that if $(\psi, \varphi)$ are conjugate with respect to the cost $c_{n}$, then by Lemma 6.1 they are both Lipschitz continuous with Lipschitz constant less than the Lipschitz constant of $c_{n}$.

Let us define:

$$
\Delta:=\left\{(\mu, \nu) \in C^{0}\left(\bar{\Omega}, \mathbb{R}_{++}\right)^{2}, \int_{\Omega} \mu d x=\int_{\Omega} \nu d y\right\}
$$

We will prove existence of $\varepsilon$-equilibria for the $\operatorname{cost} c_{n}$ by reformulating the problem as a fixed-point problem for some operator on some subset of $\Delta$ and using Schauder's fixed-point Theorem. We will then prove existence of $\varepsilon$-equilibria for the cost $c$ by letting $n$ tend to $+\infty$. 


\subsubsection{Deducing wages and revenues from densities}

Theorem 6.1 and proposition 6.1 are of particular interest in our equilibrium problem since they imply that wages and revenues can be deduced from densities. Indeed, those results from optimal transportation imply that, given a continuous positive density of residents $\mu$, and a continuous positive density of employment $\nu$, with same total mass there is a unique (up to the addition of a constant) pair of wage and revenue functions $\psi$ and $\varphi$ which induce a consistent transportation plan. More precisely, assume that $\psi$ and $\varphi$ are linked by the free mobility of labour conjugacy relations:

$$
\begin{aligned}
& \varphi(x)=\sup \left\{\psi(y)-c_{n}(x, y): y \in \Omega\right\} \\
& \psi(y)=\inf \left\{\varphi(x)+c_{n}(x, y): x \in \Omega\right\}
\end{aligned}
$$

and that the transportation plan $\gamma \in \Pi(\mu, \nu)$ is consistent with free-mobility of labor:

$$
\psi(y)-\varphi(x)=c_{n}(x, y) \gamma \text {-a.e.. }
$$

The previous conditions, together with Theorem 6.1 mean that $(\psi, \varphi)$ is a solution of:

$$
\left(\mathcal{D}_{\mu, \nu}^{n}\right) \sup _{\psi, \varphi}\left\{\int_{\bar{\Omega}} \psi d \nu-\int_{\bar{\Omega}} \varphi d \mu: \psi(y)-\varphi(x) \leq c_{n}(x, y),(x, y) \in \Omega^{2}\right\}
$$

and $\gamma$ is an optimal transportation plan between $\mu$ and $\nu$ for the cost $c_{n}$. Finally, since $c_{n}$ is smooth, proposition 6.1 shows that $\left(\mathcal{D}_{\mu, \nu}^{n}\right)$ admits a unique solution $(\psi, \varphi)$ up to the addition of the same constant to both functions. Let us also remark that $(\psi, \varphi)$ satisfies the conjugacy relations (with respect to the regularized $\operatorname{cost} c_{n}$ ). If we impose the additional normalization condition $\inf _{\Omega} \psi=1$, this defines wages and revenues as functions of densities $(\mu, \nu) \in$ $\Delta$.

For fixed $(\mu, \nu) \in \Delta$, define a wage function $\psi$ and a revenue function $\varphi$ as the unique solution of the program $\left(\mathcal{D}_{\mu, \nu}^{n}\right)$ which satisfies the normalization condition $\inf _{\Omega} \psi=1$ and set $(\psi, \varphi)=T_{1}^{n}(\mu, \nu)$. Let us remark that by Ascoli's theorem, Lemma 6.1, our normalization condition and the conjugacy relations, the set $T_{1}^{n}(\Delta)$ is included in some compact subset (independent of both $\varepsilon$ and $n$ ) of $C^{0}(\bar{\Omega}, \mathbb{R})^{2}$ for the uniform topology. More precisely, the following holds:

Lemma 8.1 Let $(\psi, \varphi) \in T_{1}^{n}(\Delta)$, then:

- for all $\left(x_{1}, x_{2}, y_{1}, y_{2}\right) \in \bar{\Omega}^{4}$, one has:

$$
\left|\varphi\left(x_{1}\right)-\varphi\left(x_{2}\right)\right| \leq \omega_{1}\left(\left|x_{1}-x_{2}\right|\right),\left|\psi\left(y_{1}\right)-\psi\left(y_{2}\right)\right| \leq \omega_{2}\left(\left|y_{1}-y_{2}\right|\right) .
$$


- $1+\omega_{2}(\operatorname{diam}(\bar{\Omega})) \geq \varphi \geq \psi \geq 1$ on $\bar{\Omega}$.

Proof: The first assertion follows from Lemma 6.1, it implies in particular $\sup _{\bar{\Omega}} \psi-\inf _{\bar{\Omega}} \psi \leq \omega_{2}(\operatorname{diam}(\bar{\Omega}))$. Since $c_{n}(x, x)=0$, from the conjugacy relations, we have $\varphi \geq \psi \geq 1$ and since $c_{n} \geq 0$ we get $\varphi \leq \sup _{\bar{\Omega}} \psi \leq$ $1+\omega_{2}(\operatorname{diam}(\bar{\Omega}))$.

\subsubsection{Deducing densities from wages and revenues}

Given densities $\mu$ and $\nu$ as before, so that they have the same total mass, assume now that we are given the wage function $\psi$ and the revenue function $\varphi$ satisfying the normalization $\min \psi=1$ as in the previous paragraph. We now aim to prove that there exists a unique constant $\lambda>-1$ (so that $\psi+\lambda$ and $\varphi+\lambda$ remain positive) such that:

$$
\int_{\Omega} \widetilde{\mu}_{\varepsilon}\left(Z_{\nu}(x), \psi(x)+\lambda, \varphi(x)+\lambda\right) d x=\int_{\Omega} \widetilde{\nu}_{\varepsilon}\left(Z_{\nu}(x), \psi(x)+\lambda, \varphi(x)+\lambda\right) d x .
$$

So $\widetilde{\mu}_{\varepsilon}\left(Z_{\nu}(x), \psi(x)+\lambda, \varphi(x)+\lambda\right)$ and $\widetilde{\nu}_{\varepsilon}\left(Z_{\nu}(x), \psi(x)+\lambda, \varphi(x)+\lambda\right)$ will be a new density of residents and a new density of jobs, still having same total mass.

Proposition 8.1 Let $\varepsilon \in(0,1 / 2), n \geq r^{-1},(\mu, \nu) \in \Delta$ and $(\psi, \varphi):=$ $T_{1}^{n}(\mu, \nu)$. The equation

$$
\int_{\Omega} \widetilde{\mu}_{\varepsilon}\left(Z_{\nu}(x), \psi(x)+\lambda, \varphi(x)+\lambda\right) d x=\int_{\Omega} \widetilde{\nu}_{\varepsilon}\left(Z_{\nu}(x), \psi(x)+\lambda, \varphi(x)+\lambda\right) d x .
$$

admits a unique root $\lambda \in(-1,+\infty)$. This root satisfies $\lambda \in\left[\lambda_{1}, \lambda_{2}\right]$, where $\lambda_{2} \geq \lambda_{1}>-1$ and those two constants do not depend on $(\mu, \nu, \varepsilon, n)$.

Proof: For $\lambda>-1$ define:

$$
\begin{aligned}
& F_{\varepsilon}(\lambda):=\int_{\Omega} \widetilde{\mu}_{\varepsilon}\left(Z_{\nu}(x), \psi(x)+\lambda, \varphi(x)+\lambda\right) d x \\
& G_{\varepsilon}(\lambda):=\int_{\Omega} \widetilde{\nu}_{\varepsilon}\left(Z_{\nu}(x), \psi(x)+\lambda, \varphi(x)+\lambda\right) d x
\end{aligned}
$$

Because of assumptions 3 and $4, F_{\varepsilon}$ is continuous increasing and $G_{\varepsilon}$ is continuous decreasing on $(-1,+\infty)$. Using assumptions 3 and 4 again, and since $\varphi \geq \psi \geq 1$ and $\bar{z} \geq Z_{\nu} \geq \underline{z}$ on $\Omega$, we first find that:

$$
F_{\varepsilon}(\lambda) \geq \widetilde{F}(\lambda):=|\Omega| N(1+\lambda)\left(1-\theta_{\varepsilon}(\bar{z}, 1+\lambda, 1+\lambda)\right)
$$


By definition of $\theta_{\varepsilon}$ and using assumptions 3,4 and 6, there exists $\alpha \in$ $(-1,+\infty)$ such that $\theta_{\varepsilon}(\bar{z}, 1+\lambda, 1+\lambda) \leq 1 / 2$ for all $\lambda \geq \alpha$ and all $\varepsilon \in(0,1 / 2)$. Hence for $\lambda \geq \alpha$, (8.8) yields:

$$
F_{\varepsilon}(\lambda) \geq \widetilde{F}(\lambda) \geq \frac{|\Omega|}{2} N(1+\lambda)
$$

Similarly, one gets:

$$
G_{\varepsilon}(\lambda) \leq \widetilde{G}(\lambda):=|\Omega| \cdot n(\bar{z}, 1+\lambda)
$$

Note that $\widetilde{F}$ is increasing and $\widetilde{G}$ decreasing, that both are obviously continuous, and by (8.9) and assumption 3, we have:

$$
\begin{array}{r}
(\widetilde{F}-\widetilde{G})(\lambda) \geq|\Omega|(N(1+\lambda)-n(\bar{z}, 1+\lambda)) \text { for } \lambda \geq \alpha \\
\lim _{\lambda \rightarrow+\infty}(N(1+\lambda)-n(\bar{z}, 1+\lambda))=+\infty .
\end{array}
$$

Hence there exists a constant $\lambda_{2} \in(\alpha,+\infty)$, that does not depend on $(\mu, \nu, \varepsilon, n)$, such that on $\left(\lambda_{2},+\infty\right)$, one has

$$
F_{\varepsilon}-G_{\varepsilon} \geq \widetilde{F}-\widetilde{G}>0 .
$$

Using Lemma 8.1, setting $c_{1}:=1+\omega_{2}(\operatorname{diam}(\bar{\Omega}))$, for all $x \in \Omega$, we have

$$
1 \leq \psi(x) \leq \varphi(x) \leq c_{1}
$$

Using assumption 3, we first get:

$$
F_{\varepsilon}(\lambda) \leq \bar{F}(\lambda):=|\Omega| N\left(c_{1}+\lambda\right)
$$

Note that $\bar{F}$ is an increasing function of $\lambda$. Let $y_{0} \in \bar{\Omega}$ be such that $\psi\left(y_{0}\right)=$ $1=\min \psi$. Using Lemma 8.1 again, we also get:

$$
\psi(y) \leq 1+\omega_{2}\left(\left|y-y_{0}\right|\right), \forall y \in \Omega .
$$

With assumptions 3 and 4, we obtain then:

$$
G_{\varepsilon}(\lambda) \geq \int_{\Omega} \widetilde{\nu}_{\varepsilon}\left(\underline{z}, 1+\lambda+\omega_{2}\left(\left|y-y_{0}\right|\right), c_{1}+\lambda\right) d y
$$

Using assumption 1, we then get:

$$
G_{\varepsilon}(\lambda) \geq \bar{G}(\lambda)
$$


where $\bar{G}$ is the function defined by:

$$
\bar{G}(\lambda):=\frac{\varepsilon_{0}}{\pi} \int_{B\left(0, \delta_{0}\right)} \theta_{\varepsilon}\left(\underline{z}, 1+\lambda+\omega_{2}(|x|), c_{1}+\lambda\right) n\left(\underline{z}, 1+\lambda+\omega_{2}(|x|)\right) d x
$$

By definition of $\theta_{\varepsilon}$ and using assumptions 3,4 and 6 , we deduce that there exists $\eta \in(0,2)$ such that for every $\varepsilon \in(0,1 / 2)$ and all $t \in(0, \eta]$ one has $\theta_{\varepsilon}\left(\underline{z}, t, c_{1}\right) \geq 1 / 2$. Now let $\delta \leq \delta_{0}$ be such that $\omega_{2}(\delta) \leq \eta / 2$, for all $\lambda \in(-1,-1+\eta / 2]$, one has:

$$
\bar{G}(\lambda) \geq \underline{G}(\lambda):=\frac{\varepsilon_{0}}{2 \pi} \int_{B(0, \delta)} n\left(\underline{z}, 1+\lambda+\omega_{2}(|x|)\right) d x
$$

When $\lambda \rightarrow-1^{+}$, the integrand in (8.13), $n\left(\underline{z}, 1+\lambda+\omega_{2}(|x|)\right)$, converges monotonically to $n\left(\underline{z}, \omega_{2}(|x|)\right)$. Using assumption 5 and the monotone convergence Theorem we obtain that $\underline{G}$ (which is decreasing and does not depend on $(\mu, \nu, \varepsilon, n))$ tends to $+\infty$ as $\lambda$ tends to -1 . Hence there exists $\lambda_{1} \in(-1,-1+\eta / 2]$ such that on $\left(-1, \lambda_{1}\right)$ one has:

$$
F_{\varepsilon}-G_{\varepsilon} \leq \bar{F}-\bar{G} \leq \bar{F}-\underline{G}<0 .
$$

Finally, from (8.14), (8.11), and the monotonicity and continuity properties of $F_{\varepsilon}$ and $G_{\varepsilon}$ we deduce that the equation $F_{\varepsilon}=G_{\varepsilon}$ admits a unique root $\lambda \in(-1,+\infty)$ and $\lambda_{2} \geq \lambda \geq \lambda_{1}$. Since $\lambda_{2}$ and $\lambda_{1}$ do not depend on $(\mu, \nu, \varepsilon, n)$, we are done.

Note that with $\lambda$ determined as in the previous proposition the two functions $\psi+\lambda$ and $\varphi+\lambda$ are strictly positive, of course they still are conjugate with respect to $c_{n}$ and still solve the program $\left(\mathcal{D}_{\mu, \nu}^{n}\right)$.

Besides, under our continuity assumptions, we have obtained the two new densities $\left(\mu^{\prime}, \nu^{\prime}\right) \in \Delta$ :

$$
\begin{aligned}
\mu^{\prime} & :=\widetilde{\mu}_{\varepsilon}\left(Z_{\nu}(x), \psi(x)+\lambda, \varphi(x)+\lambda\right) \\
\nu^{\prime} & :=\widetilde{\nu}_{\varepsilon}\left(Z_{\nu}(x), \psi(x)+\lambda, \varphi(x)+\lambda\right) .
\end{aligned}
$$

\subsubsection{Fixed-point formulation}

In what follows, we assume $\varepsilon \in(0,1 / 2), n \geq r^{-1}$. We sum up the preceding results by showing that the existence of $\varepsilon$-equilibria for the regularized transportation cost $c_{n}$ reduces to a certain fixed-point problem for an operator $T^{\varepsilon, n}: \Delta \rightarrow \Delta$ which we construct as follows.

Start from $(\mu, \nu) \in \Delta$, a pair of densities. 


\section{Step 1:}

Define the productivity function $z$ by : $z=Z_{\nu}$. In view of formula (2.1), it is immediate to check that $z \in C^{0}(\bar{\Omega},[\underline{z}, \bar{z}])$.

\section{Step 2:}

Let $(\psi, \varphi)$ be the only solution of problem $\left(\mathcal{D}_{\mu, \nu}^{n}\right)$ such that $\min _{\bar{\Omega}} \psi=1$. Denote $(\psi, \varphi):=T_{1}^{n}(\mu, \nu)$. Notice that $\psi, \varphi$ are continuous functions and that $\varphi \geq \psi \geq 1$.

\section{Step 3:}

Find the only constant $\lambda>-1$ such that:

$$
\int_{\Omega} \widetilde{\mu}_{\varepsilon}\left(Z_{\nu}(x), \psi(x)+\lambda, \varphi(x)+\lambda\right) d x=\int_{\Omega} \widetilde{\nu}_{\varepsilon}\left(Z_{\nu}(x), \psi(x)+\lambda, \varphi(x)+\lambda\right) d x
$$

and define $(\psi+\lambda, \varphi+\lambda):=T_{2}^{\varepsilon}\left(\psi, \varphi, Z_{\nu}\right)$. Proposition 8.1 guarantees that $T_{2}^{\varepsilon}\left(\psi, \varphi, Z_{\nu}\right)$ is well-defined and $T_{2}^{\varepsilon}\left(\psi, \varphi, Z_{\nu}\right)$ is a pair of strictly positive continuous functions on $\bar{\Omega}$.

\section{Step 4:}

Compute the new element $\left(\mu^{\prime}, \nu^{\prime}\right)$ of $\Delta$, defined for all $x \in \Omega$ by:

$$
\begin{aligned}
\mu^{\prime}(x): & =\widetilde{\mu}_{\varepsilon}\left(Z_{\nu}(x), \psi(x)+\lambda, \varphi(x)+\lambda\right)=\widetilde{\nu}_{\varepsilon}\left(Z_{\nu}(x), T_{2}^{\varepsilon}\left(T_{1}^{n}(\mu, \nu), Z_{\nu}\right)(x)\right) \\
\nu^{\prime}(x): & =\widetilde{\nu}_{\varepsilon}\left(Z_{\nu}(x), \psi(x)+\lambda, \varphi(x)+\lambda\right)=\widetilde{\nu}_{\varepsilon}\left(Z_{\nu}(x), T_{2}^{\varepsilon}\left(T_{1}^{n}(\mu, \nu), Z_{\nu}\right)(x)\right)
\end{aligned}
$$

and denote by $T^{\varepsilon, n}(\mu, \nu):=\left(\mu^{\prime}, \nu^{\prime}\right)$ this new pair of densities. It is immediate to check that by construction $T^{\varepsilon, n}(\mu, \nu) \in \Delta$.

Existence of an $\varepsilon$-equilibrium for the transportation cost $c_{n}$ actually reduces to the existence of fixed-points of $T^{\varepsilon, n}$. This is the content of the next result:

Proposition 8.2 Let $(\mu, \nu) \in \Delta,(\psi, \varphi):=T_{2}^{\varepsilon}\left(T_{1}^{n}(\mu, \nu), Z_{\nu}\right)$ and let $\gamma \in$ $\Pi(\mu, \nu)$ be an optimal transportation plan between $\mu$ and $\nu$ for the transportation cost $c_{n}$. The next statement are equivalent:

1. $(\mu, \nu)$ is a fixed-point of $T^{\varepsilon, n}$,

2. $\left(\mu, \nu, Z_{\nu}, \psi, \varphi, \gamma\right)$ is an $\varepsilon$-equilibrium for the transportation cost $c_{n}$.

Proof: Assume first that $(\mu, \nu) \in \Delta$ and $(\mu, \nu)=T^{\varepsilon, n}(\mu, \nu)$. By definition, requirements 1 and 2 in the definition of an $\varepsilon$-equilibrium are satisfied. The conjugacy requirement 4 follows from Theorem 6.1, Lemma 6.1 and the definition of $T_{1}^{n}$ and $T_{2}^{\varepsilon}$. Requirement 5 follows from Theorem 6.1 and the fact 
that $(\psi, \varphi)$ solves $\left(\mathcal{D}_{\mu, \nu}^{n}\right)$. Finally since $(\mu, \nu)$ is a fixed-point of $T^{\varepsilon, n}$ and by definition of $(\psi, \varphi)$, we have:

$$
\mu(.)=\widetilde{\mu}_{\varepsilon}\left(Z_{\nu}(.), \psi(.), \varphi(.)\right), \nu(.)=\widetilde{\nu}_{\varepsilon}\left(Z_{\nu}(.), \psi(.), \varphi(.)\right)
$$

so that requirement 3 is satisfied. The converse implication follows from requirement 3 in the definition of an $\varepsilon$-equilibrium and the definition of $T^{\varepsilon, n}$.

\subsubsection{Existence of $\varepsilon$-equilibria in the smooth case}

We shall prove here that the operator $T^{\varepsilon, n}$ satisfies the assumptions of Schauder's fixed-point Theorem, on some closed convex subset of $\Delta$ so that it admits at least one fixed-point.

Lemma 8.2 Let $\varepsilon \in(0,1 / 2)$ and $n \geq r^{-1}$. Under assumptions 1, 2, 3, 4, 5, 6 , there exist three positive constants $\alpha_{1}, \alpha_{2}, \alpha_{3}$ such that:

$$
T^{\varepsilon, n}(\Delta) \subset \Delta_{\varepsilon \alpha_{1}, \alpha_{2}, \alpha_{3}}:=\left\{\left(\mu^{\prime}, \nu^{\prime}\right) \in C^{0}\left(\bar{\Omega},\left[\varepsilon \alpha_{1}, \alpha_{2}\right]\right)^{2}: \int_{\Omega} \mu^{\prime}=\int_{\Omega} \nu^{\prime} \geq \alpha_{3}\right\} .
$$

Proof: Let $(\mu, \nu) \in \Delta$ and $\left(\mu^{\prime}, \nu^{\prime}\right):=T^{\varepsilon, n}(\mu, \nu)$. Defining $z:=Z_{\nu}$ and $(\psi, \varphi):=T_{2}^{\varepsilon}\left(T_{1}^{n}(\mu, \nu), Z_{\nu}\right)$, we then have, for all $x \in \bar{\Omega}$ :

$$
\begin{gathered}
\mu^{\prime}(x)=\left(1-\theta_{\varepsilon}(z(x), \psi(x), \varphi(x))\right) N(\varphi(x)) \\
\nu^{\prime}(x)=\theta_{\varepsilon}(z(x), \psi(x), \varphi(x)) n(z(x), \psi(x))
\end{gathered}
$$

By construction, $\varepsilon \leq \theta_{\varepsilon}(., .,.) \leq 1-\varepsilon$ and $\underline{z} \leq z(.) \leq \bar{z}$. Using Lemma 8.1 and proposition 8.1, setting $C_{0}=1+\lambda_{1}>0$ and $C_{1}:=1+\lambda_{2}+\omega_{2}(\operatorname{diam}(\bar{\Omega}))$, one gets:

$$
C_{0} \leq \psi \leq C_{1} \text {, and } C_{0} \leq \varphi \leq C_{1} \text { on } \bar{\Omega} .
$$

Using (8.15), assumptions 3 and 4 , we then obtain, for all $x \in \bar{\Omega}$ :

$$
\begin{array}{r}
N\left(C_{0}\right) \leq N(\varphi(x)) \leq N\left(C_{1}\right) \\
n\left(\underline{z}, C_{1}\right) \leq n(z(x), \psi(x)) \leq n\left(\bar{z}, C_{0}\right) .
\end{array}
$$

Defining:

$$
\alpha_{1}:=\min \left(N\left(C_{0}\right), n\left(\underline{z}, C_{1}\right)\right), \alpha_{2}:=\max \left(N\left(C_{1}\right), n\left(\bar{z}, C_{0}\right)\right),
$$

we therefore have $\left(\mu^{\prime}, \nu^{\prime}\right) \in C^{0}\left(\bar{\Omega},\left[\varepsilon \alpha_{1}, \alpha_{2}\right]\right)^{2}$. Finally, the fact that $\mu^{\prime}$ and $\nu^{\prime}$ have the same total mass yields:

$$
\int_{\Omega} \theta_{\varepsilon}(z(x), \psi(x), \varphi(x))(n(z(x), \psi(x))+N(\varphi(x))) d x=\int_{\Omega} N(\varphi(x)) d x
$$


since $n(z(x), \psi(x))+N(\varphi(x)) \leq n\left(\bar{z}, C_{0}\right)+N\left(C_{1}\right)$ and $N(\varphi(x)) \geq N\left(C_{0}\right)$ for all $x \in \bar{\Omega}$, we get:

$$
\int_{\Omega} \theta_{\varepsilon}(z(x), \psi(x), \varphi(x)) d x \geq \frac{|\Omega| N\left(C_{0}\right)}{n\left(\bar{z}, C_{0}\right)+N\left(C_{1}\right)}
$$

hence:

$$
\int_{\Omega} \mu^{\prime}=\int_{\Omega} \nu^{\prime} \geq \alpha_{3}=\frac{|\Omega| N\left(C_{0}\right) n\left(\underline{z}, C_{1}\right)}{n\left(\bar{z}, C_{0}\right)+N\left(C_{1}\right)}
$$

In what follows, $C^{0}(\bar{\Omega}, \mathbb{R})^{2}$ will be equipped with the topology of uniform convergence, i.e. with the norm:

$$
\left\|\left(\mu_{1}, \nu_{1}\right)-\left(\mu_{2}, \nu_{2}\right)\right\|_{\infty}:=\sup _{x \in \bar{\Omega}}\left|\mu_{1}(x)-\mu_{2}(x)\right|+\sup _{y \in \bar{\Omega}}\left|\nu_{1}(y)-\nu_{2}(y)\right| .
$$

Note then that $\Delta_{\varepsilon \alpha_{1}, \alpha_{2}, \alpha_{3}}$ is a closed convex subset of $C^{0}(\bar{\Omega}, \mathbb{R})^{2}$.

Lemma 8.3 Let $\varepsilon \in(0,1 / 2)$ and $n \geq r^{-1}$. Under assumptions 1, 2, 3, 4, 5, $6, T^{\varepsilon, n}$ is a continuous mapping on $\Delta_{\varepsilon \alpha_{1}, \alpha_{2}, \alpha_{3}}$ equipped wih the norm $\|.\|_{\infty}$.

Proof: Let $(\mu, \nu) \in \Delta_{\varepsilon \alpha_{1}, \alpha_{2}, \alpha_{3}}$ and $\left(\mu_{k}, \nu_{k}\right)$ be a sequence of elements of $\Delta_{\varepsilon \alpha_{1}, \alpha_{2}, \alpha_{3}}$ converging to $(\mu, \nu)$. Define then:

$$
z_{k}:=Z_{\nu_{k}},\left(\psi_{k}, \varphi_{k}\right):=T_{2}^{\varepsilon}\left(T_{1}^{n}\left(\mu_{k}, \nu_{k}\right), z_{k}\right) .
$$

From lemma 8.5 , we know that $z_{k}$ converges uniformly to $Z_{\nu}$ on $\bar{\Omega}$. Using lemma 8.1, proposition 8.1 and Ascoli's Theorem, $\left(\psi_{k}, \varphi_{k}\right)$ admits a converging subsequence that we denote $\left(\psi_{k_{j}}, \varphi_{k_{j}}\right)$ and we denote $(\psi, \varphi)$ its limit. Note that by proposition 8.1, $\psi$ and $\varphi$ are strictly positive on $\bar{\Omega}$. By definition of $T^{\varepsilon, n}$ and proposition 6.1 , for all $k,\left(\psi_{k}, \varphi_{k}\right)$ is characterized by the conditions:

$$
\int_{\Omega} \widetilde{\mu}_{\varepsilon}\left(z_{k}(x), \psi_{k}(x), \varphi_{k}(x)\right) d x=\int_{\Omega} \widetilde{\nu}_{\varepsilon}\left(z_{k}(x), \psi_{k}(x), \varphi_{k}(x)\right) d x
$$

and $\left(\psi_{k}, \varphi_{k}\right)$ solves the optimization problem:

$$
\left(\mathcal{D}_{\mu_{k}, \nu_{k}}^{n}\right) \sup _{\psi, \varphi}\left\{\int_{\bar{\Omega}} \psi d \nu_{k}-\int_{\bar{\Omega}} \varphi d \mu_{k}: \psi(y)-\varphi(x) \leq c_{n}(x, y), \quad(x, y) \in \Omega^{2}\right\} .
$$


It is easy to see, letting $j$ go to $\infty$, that $(\psi, \varphi)$ solves:

$$
\left(\mathcal{D}_{\mu, \nu}^{n}\right) \sup _{\psi, \varphi}\left\{\int_{\bar{\Omega}} \psi d \nu-\int_{\bar{\Omega}} \varphi d \mu: \psi(y)-\varphi(x) \leq c_{n}(x, y),(x, y) \in \Omega^{2}\right\} .
$$

Passing to the limit in (8.18), yields:

$$
\int_{\Omega} \widetilde{\mu}_{\varepsilon}\left(Z_{\nu}(x), \psi(x), \varphi(x)\right) d x=\int_{\Omega} \widetilde{\nu}_{\varepsilon}\left(Z_{\nu}(x), \psi(x), \varphi(x)\right) d x
$$

Together with (8.20) and propositions 6.1 and 8.1, this implies $(\psi, \varphi)=$ $T_{2}^{\varepsilon}\left(T_{1}^{n}(\mu, \nu), Z_{\nu}\right)$ and that $(\psi, \varphi)$ is the only cluster point of the sequence $\left(\psi_{k}, \varphi_{k}\right)$. Hence, by compactness, the whole sequence $\left(\psi_{k}, \varphi_{k}\right)$ converges uniformly to $(\psi, \varphi)$ on $\bar{\Omega}$. Finally, by continuity of $\widetilde{\mu}_{\varepsilon}(., .,$.$) and \widetilde{\nu}_{\varepsilon}(., .,$.$) ,$ $T^{\varepsilon, n}\left(\mu_{k}, \nu_{k}\right)$ converges uniformly to $T^{\varepsilon, n}(\mu, \nu)$ on $\bar{\Omega}$

Lemma 8.4 Let $\varepsilon \in(0,1 / 2)$ and $n \geq r^{-1}$. Under assumptions 1, 2, 3, 4, 5, $6, T^{\varepsilon, n}\left(\Delta_{\varepsilon \alpha_{1}, \alpha_{2}, \alpha_{3}}\right)$ is relatively compact in $C^{0}(\bar{\Omega}, \mathbb{R})^{2}$ equipped with the norm $\|\cdot\|_{\infty}$.

Proof: Let $\left(\mu_{k}, \nu_{k}\right)$ be a sequence of elements of $\Delta_{\varepsilon \alpha_{1}, \alpha_{2}, \alpha_{3}}$ and define $\left(\mu_{k}^{\prime}, \nu_{k}^{\prime}\right):=T^{\varepsilon, n}\left(\mu_{k}, \nu_{k}\right)$. Define then:

$$
z_{k}:=Z_{\nu_{k}},\left(\psi_{k}, \varphi_{k}\right):=T_{2}^{\varepsilon}\left(T_{1}^{n}\left(\mu_{k}, \nu_{k}\right), z_{k}\right) .
$$

Arguing as in the proof of lemma 8.3, we deduce that $\left(\psi_{k}, \varphi_{k}\right)$ admits a converging subsequence that we denote $\left(\psi_{k_{j}}, \varphi_{k_{j}}\right)$ and we denote $(\psi, \varphi)$ its limit. From lemma 8.6, we may also assume that $z_{k_{j}}$ converges to some function $z$. By continuity of $\widetilde{\mu}_{\varepsilon}(., .,$.$) and \widetilde{\nu}_{\varepsilon}(., . .$.$) , we deduce that \mu_{k_{j}}^{\prime}$ and $\nu_{k_{j}}^{\prime}$ converge uniformly on $\bar{\Omega}$ respectively to $\widetilde{\mu}_{\varepsilon}(z(),. \psi(),. \varphi()$.$) and \widetilde{\nu}_{\varepsilon}(z(),. \psi(),. \varphi()$.$) .$

Combining proposition 8.2, with lemmas 8.2, 8.3, 8.4 and invoking Schauder's fixed-point Theorem, we finally obtain the existence of $\varepsilon$-equilibria for the transportation cost $c_{n}$ :

Theorem 8.1 Let $\varepsilon \in(0,1 / 2)$ and $n \geq r^{-1}$. Under assumptions 1, 2, 3, 4, 5,6 , there exists at least one $\varepsilon$-equilibrium for the transportation cost $c_{n}$.

Proof: From lemmas 8.2, 8.3, 8.4, the continuous operator $T^{\varepsilon, n}$ maps the closed convex set $\Delta_{\varepsilon \alpha_{1}, \alpha_{2}, \alpha_{3}}$ into itself and has a relatively compact image. By Schauder's fixed-point Theorem (see for instance [5]), $T^{\varepsilon, n}$ admits at least one fixed-point on $\Delta_{\varepsilon \alpha_{1}, \alpha_{2}, \alpha_{3}}$. Using proposition 8.2, this implies that there exists at least one $\varepsilon$-equilibrium for the transportation cost $c_{n}$. 


\subsubsection{Existence of $\varepsilon$-equilibria when $c$ is continuous}

Letting the mollifying parameter $n$ tend to $\infty$ (for fixed $\varepsilon>0$ ) and using theorem 8.1, we then get existence of $\varepsilon$-equilibria:

Theorem 8.2 Let $\varepsilon \in(0,1 / 2)$. Under assumptions 1, 2, 3, 4, 5, 6, there exists at least one $\varepsilon$-equilibrium for the transportation cost $c$.

Proof: From the results of the previous paragraphs, for every $n \geq r^{-1}$, there exists an $\varepsilon$-equilibrium for the transport $\operatorname{cost} c_{n}$ that is of the form $\left(\mu_{n}, \nu_{n}, z_{n}, \psi_{n}, \varphi_{n}, \gamma_{n}\right)$ with $\left(\mu_{n}, \nu_{n}\right)$ a fixed-point of $T^{\varepsilon, n}$ on $\Delta_{\varepsilon \alpha_{1}, \alpha_{2}, \alpha_{3}}, z_{n}:=$ $Z_{\nu_{n}},\left(\psi_{n}, \varphi_{n}\right)=T_{2}^{\varepsilon}\left(T_{1}^{n}\left(\mu_{n}, \nu_{n}\right), z_{n}\right)$ and $\gamma_{n}$ an optimal transportation plan between $\mu_{n}$ and $\nu_{n}$ for the cost $c_{n}$. Using lemma 8.1, proposition 8.1 and Ascoli's Theorem, $\left(\psi_{n}, \varphi_{n}\right)$ admits a converging subsequence, that we do not relabel, and we denote $(\psi, \varphi)$ its limit (note that $\psi$ and $\varphi$ are strictly positive on $\bar{\Omega}$ by proposition 8.1). By lemma 8.6, we may also assume that $z_{n}$ converges uniformly to some function $z$ on $\bar{\Omega}$. By continuity of $\widetilde{\mu}_{\varepsilon}(., .,$.$) and$ $\widetilde{\nu}_{\varepsilon}(., .,$.$) , we have uniform convergence of \mu_{n}$ and $\nu_{n}$ to $\mu:=\widetilde{\mu}_{\varepsilon}(z(),. \psi(),. \varphi()$. and $\nu:=\widetilde{\nu}_{\varepsilon}(z(),. \psi(),. \varphi()$.$) respectively. Note that (\mu, \nu) \in \Delta_{\varepsilon \alpha_{1}, \alpha_{2}, \alpha_{3}}$. With lemma 8.5, we also have $z=Z_{\nu}$.

Due to lemma $8.7,(\psi, \varphi)$ are conjugate with respect to the cost $c$, i.e. fulfill (8.2) and (8.3). For all $n, \gamma_{n} \in \Pi\left(\mu_{n}, \nu_{n}\right)$ is a nonnegative Borel measure on $\bar{\Omega} \times \bar{\Omega}$ and $\gamma_{n}(\bar{\Omega} \times \bar{\Omega}) \leq \alpha_{2}|\Omega|$ by lemma 8.2 . We may therefore assume that $\gamma_{n}$ converges weakly $*$ to some measure $\gamma$. By weak ${ }^{*}$ convergence of marginals, we must have $\gamma \in \Pi(\mu, \nu)$. Finally, passing to the limit in the identity:

$$
\int_{\bar{\Omega} \times \bar{\Omega}} c_{n} d \gamma_{n}=\int_{\bar{\Omega}} \psi_{n} d \nu_{n}-\int_{\bar{\Omega}} \varphi_{n} d \mu_{n}=\int_{\bar{\Omega} \times \bar{\Omega}}\left(\psi_{n}(y)-\varphi_{n}(x)\right) d \gamma_{n}(x, y)
$$

and using $\psi-\varphi \leq c$, we get:

$$
\psi(y)-\varphi(x)=c(x, y) \gamma \text {-a.e.. }
$$

We thus have proven that $(\mu, \nu, z, \psi, \varphi, \gamma)$ is an $\varepsilon$-equilibrium for the transportation cost $c$.

Let us remark that the $\varepsilon$-equilibrium $(\mu, \nu, z, \psi, \varphi, \gamma)$ we have obtained in the previous proof satisfies: $(\mu, \nu) \in \Delta_{\varepsilon \alpha_{1}, \alpha_{2}, \alpha_{3}}, z \in Z\left(C^{0}\left(\bar{\Omega},\left[0, \alpha_{2}\right]\right)\right.$. Moreover, by lemmas 8.2 and $6.1, \psi$ and $\varphi$ belong to some uniformly equicontinuous subset of $C^{0}\left(\bar{\Omega},\left[C_{0}, C_{1}\right]\right)$ for some positive constants $C_{0}$ and $C_{1}$ that do not depend on $\varepsilon$. By Ascoli's theorem and lemma 8.6, we deduce that $(z, \psi, \varphi)$ belong to some compact subset (that does not depend on $\varepsilon$ ) of $C^{0}(\bar{\Omega},[\underline{z}, \bar{z}]) \times C^{0}\left(\bar{\Omega},\left[C_{0}, C_{1}\right]\right) \times C^{0}\left(\bar{\Omega},\left[C_{0}, C_{1}\right]\right)$ equipped with the uniform topology 


\subsection{From $\varepsilon$-equilibria to equilibria: proof of theorem 6.2}

From the results of subsection 8.2.5, for every $\varepsilon \in(0,1 / 2)$, there exists an $\varepsilon$-equilibrium for the transportation cost $c,\left(\mu_{\varepsilon}, \nu_{\varepsilon}, z_{\varepsilon}, \psi_{\varepsilon}, \varphi_{\varepsilon}, \gamma_{\varepsilon}\right)$ such that $\left(\mu_{\varepsilon}, \nu_{\varepsilon}\right) \in \Delta_{\varepsilon \alpha_{1}, \alpha_{2}, \alpha_{3}}$ and the family $\left(z_{\varepsilon}, \psi_{\varepsilon}, \varphi_{\varepsilon}\right)$ is relatively compact in $C^{0}(\bar{\Omega},[\underline{z}, \bar{z}]) \times$ $C^{0}\left(\bar{\Omega},\left[C_{0}, C_{1}\right]\right) \times C^{0}\left(\bar{\Omega},\left[C_{0}, C_{1}\right]\right)$ equipped with the uniform topology where $C_{0}$ and $C_{1}$ are positive constants (see lemma 8.2). There exists then $\varepsilon_{k}$, a sequence converging to 0 as $k$ tends to $\infty$, and $(z, \psi, \varphi) \in C^{0}(\bar{\Omega},[\underline{z}, \bar{z}]) \times$ $C^{0}\left(\bar{\Omega},\left[C_{0}, C_{1}\right]\right) \times C^{0}\left(\bar{\Omega},\left[C_{0}, C_{1}\right]\right)$ such that $\left(z_{\varepsilon_{k}}, \psi_{\varepsilon_{k}}, \varphi_{\varepsilon_{k}}\right)$ converges uniformly on $\bar{\Omega}$ to $(z, \psi, \varphi)$. For notational conveniency we shall simply relabel quantities as follows:

$$
\left(\mu_{k}, \nu_{k}, z_{k}, \psi_{k}, \varphi_{k}, \gamma_{k}\right):=\left(\mu_{\varepsilon_{k}}, \nu_{\varepsilon_{k}}, z_{\varepsilon_{k}}, \psi_{\varepsilon_{k}}, \varphi_{\varepsilon_{k}}, \gamma_{\varepsilon_{k}}\right)
$$

Let us also define:

$$
\theta_{k}:=\theta_{\varepsilon_{k}}\left(z_{k}, \psi_{k}, \varphi_{k}, \gamma_{k}\right), N_{k}:=N\left(\varphi_{k}\right), n_{k}:=n\left(z_{k}, \varphi_{k}\right)
$$

and $Q_{k}:=Q\left(\varphi_{k}\right), q_{k}:=q\left(z_{k}, \varphi_{k}\right)$. We then have the following uniform convergence on $\bar{\Omega}$ :

$$
\lim _{k}\left(N_{k}, n_{k}, Q_{k}, q_{k}\right)=(N(\varphi), n(z, \psi), Q(\varphi), q(z, \psi)) .
$$

Since $\theta_{k} \in[0,1]$, taking subsequences if necessary, we may also assume that $\theta_{k}$ converges weakly $*$ in $L^{\infty}(\Omega)$ to some function $\theta$ (of course, $0 \leq \theta \leq 1$ ). This implies that $\mu_{k}$ and $\nu_{k}$ converge weakly $*$ in $L^{\infty}(\Omega)$ to $\mu:=(1-\theta) N(\varphi)$ and $\nu:=\theta n(z, \psi)$ respectively. By weak $*$ convergence and lemma 8.2, we get

$$
\int_{\Omega} \mu(x) d x=\int_{\Omega} \nu(y) d y \geq \alpha_{3}>0 .
$$

Since $\nu_{k}$ converges weakly $*$ in $L^{\infty}(\Omega)$ to $\nu$, for all $x \in \Omega, \int_{\Omega} \rho(x, y) \nu_{k}(y) d y$ converges to $\int_{\Omega} \rho(x, y) \nu(y) d y$. Hence $z_{k}$ converges pointwise to $Z_{\nu}$ so that $z=Z_{\nu}$. By lemma 8.8, requirements (3.3) and (3.4) in the definition of an equilibrium are satisfied.

Since $\gamma_{k} \in \Pi\left(\mu_{k}, \nu_{k}\right), \gamma_{k}(\bar{\Omega} \times \bar{\Omega}) \leq \alpha_{2}|\Omega|$ by lemma 8.2. Taking a subsequence if necessary, we may therefore assume that $\gamma_{k}$ converges weakly * to some measure $\gamma$. By weak * convergence of marginals, we must have $\gamma \in \Pi(\mu, \nu)$. Passing to the limit in the identity:

$$
\int_{\bar{\Omega} \times \bar{\Omega}} c d \gamma_{k}=\int_{\bar{\Omega}} \psi_{k} d \nu_{k}-\int_{\bar{\Omega}} \varphi_{k} d \mu_{k}=\int_{\bar{\Omega} \times \bar{\Omega}}\left(\psi_{k}(y)-\varphi_{k}(x)\right) d \gamma_{k}(x, y)
$$


and using $\psi(y)-\varphi(x) \leq c(x, y)$ (this inequality is obtained by passing to the limit in $\left.\psi_{k}(y)-\varphi_{k}(x) \leq c_{k}(x, y)\right)$, we get:

$$
\psi(y)-\varphi(x)=c(x, y) \gamma \text {-a.e.. }
$$

To prove that $(\mu, \nu, z, \psi, \varphi, \theta, \gamma)$ is an equilibrium it remains to prove that the free-mobility of labor conditions (3.5) and (3.6) are satisfied. Note that (3.5) is equivalent to $\varphi=\widetilde{\varphi}$ on $\operatorname{spt}(\mu)$, with $\widetilde{\varphi}$ defined by:

$$
\widetilde{\varphi}(x):=\sup \{\psi(y)-c(x, y): y \in \operatorname{spt}(\nu)\} \text {. }
$$

On the one hand, we know from lemma 8.7 that $\psi$ and $\varphi$ satisfy the conjugacy relations (8.2) and (8.3). This implies $\varphi \geq \widetilde{\varphi}$ on $\bar{\Omega}$. On the other hand, using the conditional probabilities:

$$
\gamma=\int_{\Omega} P_{x}^{\gamma} d \mu(x)
$$

we get:

$$
\nu(\bar{\Omega})=\nu(\operatorname{spt}(\nu))=\int_{\bar{\Omega}} P_{x}^{\gamma}(\operatorname{spt}(\nu)) d \mu(x)=\int_{\bar{\Omega}} P_{x}^{\gamma}(\bar{\Omega}) d \mu(x) .
$$

Hence for $\mu$-a.e. $x$, we have $P_{x}^{\gamma}(\bar{\Omega} \backslash \operatorname{spt}(\nu))=0$. From (8.24), we also have, for $\mu$-a.e. $x, \varphi(x)=\psi(y)-c(x, y)$ for $P_{x}^{\gamma}$-a.e. $y$. Combining those two conditions, we obtain $\varphi \leq \widetilde{\varphi}$ hence $\varphi=\widetilde{\varphi} \mu$-a.e.. Since $\varphi$ and $\widetilde{\varphi}$ are continuous, the previous extends to $\varphi=\widetilde{\varphi}$ on $\operatorname{spt}(\mu)$.

The proof of (3.6) is similar. This finally proves that $(\mu, \nu, z, \psi, \varphi, \theta, \gamma)$ is an equilibrium.

\subsection{Auxiliary Lemmas}

In what follows $\alpha_{2}>0$ is as in Lemma 8.2:

Lemma 8.5 The mapping $Z$ :

$$
Z:\left\{\begin{array}{ccc}
C^{0}\left(\bar{\Omega},\left[0, \alpha_{2}\right]\right) & \longrightarrow & C^{0}(\bar{\Omega},[\underline{z}, \bar{z}]) \\
\nu & \longmapsto & Z_{\nu}
\end{array}\right.
$$

is continuous where $C^{0}\left(\bar{\Omega},\left[0, \alpha_{2}\right]\right)$ and $C^{0}(\bar{\Omega},[\underline{z}, \bar{z}])$ are endowed with the sup norm. 
Proof: The fact that $Z$ maps $C^{0}\left(\bar{\Omega},\left[0, \alpha_{2}\right]\right)$ into $C^{0}(\bar{\Omega},[\underline{z}, \bar{z}])$ is straightforward. First note that for $\nu$ and $\nu^{\prime}$ in $C^{0}\left(\bar{\Omega},\left[0, \alpha_{2}\right]\right)$ we have:

$$
\max _{x \in \bar{\Omega}} \int_{\Omega} \rho(x, y)\left|\nu(y)-\nu^{\prime}(y)\right| d y \leq C\left\|\nu-\nu^{\prime}\right\|_{\infty}
$$

where

$$
C=\max _{x \in \bar{\Omega}} \int_{\Omega} \rho(x, y) d y
$$

Since $g$ is uniformly continuous on $\left[0, \alpha_{2} C\right]$, for all $\varepsilon>0$ there exists $\delta>0$ such that $\left|g(t)-g\left(t^{\prime}\right)\right| \leq \varepsilon$ for all $\left(t, t^{\prime}\right) \in\left[0, \alpha_{2} C\right]^{2}$ such that $\left|t-t^{\prime}\right| \leq \delta$. This proves that if $\left\|\nu-\nu^{\prime}\right\|_{\infty} \leq \delta / C$ then $\left\|Z_{\nu}-Z_{\nu^{\prime}}\right\|_{\infty} \leq \varepsilon$.

Lemma 8.6 The set $Z\left(C^{0}\left(\bar{\Omega},\left[0, \alpha_{2}\right]\right)\right)$ is relatively compact in $C^{0}(\bar{\Omega},[\underline{z}, \bar{z}])$ endowed with the sup norm.

Proof: Define first the linear operator $K$ by:

$$
K:\left\{\begin{array}{ccc}
C^{0}\left(\bar{\Omega},\left[0, \alpha_{2}\right]\right) & \longrightarrow & C^{0}(\bar{\Omega}, \mathbb{R}) \\
\nu(.) & \longmapsto & K_{\nu}(.):=\int_{\Omega} \rho(., y) \nu(y) d y
\end{array}\right.
$$

Note first that $K$ is continuous and maps $C^{0}\left(\bar{\Omega},\left[0, \alpha_{2}\right]\right)$ into $C^{0}(\bar{\Omega},[0, \beta C])$ (see Lemma 8.5). Let us prove now that the set $K\left(C^{0}\left(\bar{\Omega},\left[0, \alpha_{2}\right]\right)\right)$ is uniformly equicontinuous. Let $\varepsilon>0$ and let $\delta>0$ be such that:

$$
\sup \left\{\left|\rho(x, y)-\rho\left(x^{\prime}, y\right)\right|:\left(x, x^{\prime}, y\right) \in \bar{\Omega}^{3},\left|x-x^{\prime}\right| \leq \delta\right\} \leq \frac{\varepsilon}{\alpha_{2}|\Omega|}
$$

then for all $\nu \in C^{0}\left(\bar{\Omega},\left[0, \alpha_{2}\right]\right)$ and all $\left(x, x^{\prime}\right) \in \bar{\Omega}^{2}$ such that $\left|x-x^{\prime}\right| \leq$ $\delta$, we have $\left|K_{\nu}(x)-K_{\nu}\left(x^{\prime}\right)\right| \leq \varepsilon$. Hence $K\left(C^{0}\left(\bar{\Omega},\left[0, \alpha_{2}\right]\right)\right)$ is uniformly equicontinuous.

Since $K\left(C^{0}\left(\bar{\Omega},\left[0, \alpha_{2}\right]\right)\right)$ is bounded and uniformly equicontinuous, by Ascoli's Theorem it is relatively compact in $C^{0}(\bar{\Omega}, \mathbb{R})$. The desired result follows from composition with the continuous function $g$.

Lemma 8.7 Assume that $c_{k}$ is a sequence of continuous cost functions on $\bar{\Omega}^{2}$ that converges uniformly to $c$ on $\bar{\Omega}^{2}$ as $k$ tends to $\infty$. Assume that, for all $k, \psi_{k}$ and $\varphi_{k}$ are continuous functions on $\bar{\Omega}$ that satisfy the conjugacy relations with respect to $c_{k}$ :

$$
\varphi_{k}(x)=\sup \left\{\psi_{k}(y)-c_{k}(x, y): y \in \Omega\right\}
$$




$$
\psi_{k}(y)=\inf \left\{\varphi_{k}(x)+c_{k}(x, y): x \in \Omega\right\}
$$

If $(\psi, \varphi)$ is a cluster point (for the uniform topology) of $\left(\psi_{k}, \varphi_{k}\right)$ then $(\psi, \varphi)$ are conjugate with respect to the cost $c$ in the sense that they satisfy (8.2) and (8.3).

Proof: $\quad$ First note that for all $k$ and $(x, y) \in \bar{\Omega}^{2}$, we have $\psi_{k}(y)-\varphi_{k}(x) \leq$ $c_{k}(x, y)$ hence $\psi(y)-\varphi(x) \leq c(x, y)$. Hence we have, for all $x \in \bar{\Omega}$ :

$$
\varphi(x) \geq \sup \{\psi(y)-c(x, y): y \in \Omega\} .
$$

By compactness and continuity arguments, there exists $y_{k} \in \bar{\Omega}$ such that $\varphi_{k}(x)=\psi_{k}\left(y_{k}\right)-c_{k}\left(x, y_{k}\right)$. Taking subsequences if necessary, we may assume that $y_{k}$ converges to some $y \in \bar{\Omega}$, we then obtain $\varphi(x)=\psi(y)-c(x, y)$. Hence:

$$
\varphi(x) \leq \sup \{\psi(y)-c(x, y): y \in \Omega\}
$$

This proves (8.2), the proof of (8.3) is similar.

Lemma 8.8 If $q(z(x), \psi(x))>Q(\varphi(x))$ then $\theta(x)=1, \mu(x)=0$ and $\nu(x)=$ $n(z(x), \psi(x))$. Similarly, if $q(z(x), \psi(x))<Q(\varphi(x))$ then $\theta(x)=0, \mu(x)=$ $N(\varphi(x))$ and $\nu(x)=0$.

Proof: Define:

$$
\Omega_{+}:=\{x \in \Omega: q(z(x), \psi(x))>Q(\varphi(x))\}
$$

and assume that $x \in \Omega_{+}$. For $k$ large enough $q\left(z_{k}(x), \psi_{k}(x)\right)-Q\left(\varphi_{k}(x)\right) \geq$ $\varepsilon_{k}-\varepsilon_{k}^{2}$ so that $\theta_{k}(x)=1-\varepsilon_{k}$ for $k$ large enough. In particular $\theta_{k}$ converges to 1 pointwise on $\Omega_{+}$and in $L^{1}\left(\Omega_{+}\right)$. Since $\theta_{k}$ converges to $\theta$ for the weak $*$ topology of $L^{\infty}\left(\Omega_{+}\right)$we have $\theta \equiv 1$ on $\Omega_{+}$. Similarly on $\Omega_{+}, \nu_{k}=\theta_{k} n\left(z_{k}, \psi_{k}\right)$ converges pointwise and in $L^{1}\left(\Omega_{+}\right)$to $n(z, \psi)$, hence $\nu(x)=n(z(x), \psi(x))$ a.e. on $\Omega_{+}$. Finally, on $\Omega_{+}, \mu_{k}=\left(1-\theta_{k}\right) N\left(\varphi_{k}\right)$ converges a.e. and in $L^{1}\left(\Omega_{+}\right)$to 0 , hence $\mu(x)=0$ a.e. on $\Omega_{+}$. The proof of the other assertion is similar.

\subsection{Proof of proposition 6.1}

Let $(\psi, \varphi)$ be a solution of $\left(\mathcal{D}_{\mu, \nu}\right)$. Due to the constraints of $\left(\mathcal{D}_{\mu, \nu}\right)$, we first have $\psi \leq \widetilde{\psi}$ where:

$$
\widetilde{\psi}(y):=\inf \{\varphi(x)+c(x, y): x \in \Omega\}
$$


By definition of $\widetilde{\psi}$, we also have $\varphi \geq \widetilde{\varphi}$ where:

$$
\widetilde{\varphi}(x):=\sup \{\widetilde{\psi}(y)-c(x, y): y \in \Omega\} .
$$

Using (8.26) and $\varphi \geq \widetilde{\varphi}$, we get:

$$
\widetilde{\psi}(y):=\inf \{\widetilde{\varphi}(x)+c(x, y): x \in \Omega\}
$$

so that the pair $(\widetilde{\psi}, \widetilde{\varphi})$ satisfies the conjugacy relations (8.2) and (8.3). By construction the pair $(\widetilde{\psi}, \widetilde{\varphi})$ satisfies the constraints of $\left(\mathcal{D}_{\mu, \nu}\right), \psi \leq \widetilde{\psi}$ and $\varphi \geq \widetilde{\varphi}$. Hence, one must have $\varphi=\widetilde{\varphi}, \psi=\widetilde{\psi}$ a.e.. In the sequel we may therefore assume that the pair $(\psi, \varphi)$ satisfies the conjugacy relations (8.2) and (8.3). By Lemma 6.1, and the regularity assumption on $c$, we deduce that $\psi$ and $\varphi$ are Lipschitz continous hence differentiable almost everywhere by Rademacher's Theorem (see [6]).

Let $\gamma$ be a solution of $\left(\mathcal{M}_{\mu, \nu}\right)$ then we have:

$$
\psi(y)-\varphi(x)=c(x, y) \gamma \text {-a.e.. }
$$

Let us write:

$$
\gamma=\int_{\Omega} P_{x}^{\gamma} d \mu(x)
$$

Let $x \in \Omega$ be a point of differentiability of $\varphi$ such that (8.29) holds for $P_{x}^{\gamma}$-a.e. $y$. For $h \in \mathbb{R}^{2}$ small enough, we then have for $P_{x}^{\gamma}$-a.e. $y$ :

$$
\begin{aligned}
\varphi(x+h) & =\varphi(x)+\nabla \varphi(x) \cdot h+o(h) \geq \psi(y)-c(x+h, y) \\
& =\psi(y)-c(x, y)-\nabla_{x} c(x, y) \cdot h+o(h)=\varphi(x)-\nabla_{x} c(x, y) \cdot h+o(h) .
\end{aligned}
$$

We then get that, for $P_{x}^{\gamma}$-a.e. $y$ :

$$
\nabla \varphi(x)=-\nabla_{x} c(x, y)
$$

Hence, we have:

$$
\nabla \varphi(x)=-\int_{\bar{\Omega}} \nabla_{x} c(x, y) d P_{x}^{\gamma}(y)
$$

Since the previous holds almost everywhere and a similar formula holds for $\psi$ we finally deduce the uniqueness result from the assumption that $\Omega$ is connected.

Let us remark that, under the assumptions of proposition 6.1, solutions of $\left(\mathcal{D}_{\mu, \nu}\right)$ necessarily satisfy the conjugacy relations (8.2) and (8.3). 


\subsection{Proof of theorem 5.1}

\section{The strictly convex case, $\eta>1$ :}

Let us check that the assumptions of theorem 6.2 are satisfied. Assumption 2 directly follows from the definition of $c$. Assumptions 3, 4 and 6 follow from the explicit expressions (5.1), (5.2), (5.3) an (5.4). As for assumption 5 , first remark that $c$ is $C^{1}$ on $\mathbb{R}^{2} \times \mathbb{R}^{2}$ hence Lipschitz on $\bar{\Omega}_{r} \times \bar{\Omega}_{r}$ then $\omega_{2}(t) \leq K t$ for some positive constant $K$. Using (5.1), we then get:

$$
n\left(\underline{z}, \omega_{2}(|h|)\right) \geq\left(\frac{\alpha \underline{z}^{\gamma}}{K}\right)^{\frac{1}{1-\alpha}}|h|^{-1 /(1-\alpha)} .
$$

Hence assumption 5 is satisfied provided $1 \geq 2(1-\alpha)$ i.e. $\alpha \geq 1 / 2$. Using theorem 6.2, we then obtain existence of equilibria. Finally, the fact that equilibria are pure follows from the fact that $c$ satisfies (7.1)-(7.2) when $\eta>1$ and from theorem 7.1.

The sublinear case, $\eta \leq 1$ :

As previously, it is enough to check that assumption 5 is satisfied. First note that when $\eta \leq 1$, the cost $c$ satisfies the triangle inequality (see [3]):

$$
c\left(x, y_{1}\right) \leq c\left(x, y_{2}\right)+c\left(y_{1}, y_{2}\right)
$$

this implies that $\omega_{2}(t) \leq M t^{\eta}$ hence:

$$
n\left(\underline{z}, \omega_{2}(|h|)\right) \geq\left(\frac{\alpha \underline{z}}{M}\right)^{\frac{1}{1-\alpha}}|h|^{-\eta /(1-\alpha)} .
$$

Hence assumption 5 is satisfied provided $\eta \geq 2(1-\alpha)$.

\subsection{Proof of proposition 7.1}

Assume by contradiction that $(\mu, \nu, z, \psi, \varphi, \theta, \gamma)$ is an equilibrium and $\mu=\nu$, in this case $\theta \in(0,1)$ hence $\operatorname{spt}(\mu)=\operatorname{spt}(\nu)=\bar{\Omega}$ which implies:

$$
q(z(x), \psi(x)))=Q(\varphi(x)) \text { for all } x \in \bar{\Omega} .
$$

Since $\mu=\nu$ and $\gamma \in \Pi(\mu, \nu)$ is an optimal plan between $\mu$ and $\nu=\mu$ and since $c$ vanishes only on the diagonal of $\bar{\Omega}^{2}, \gamma$ has to be supported on the diagonal of $\bar{\Omega}^{2}$ i.e. $\gamma=\int_{\Omega} \delta_{x} d \mu(x)\left(\delta_{x}\right.$ being the Dirac mass at $\left.x\right)$. Using the fact that $\varphi$ is differentiable a.e. and formula (8.31), we get, for a.e. $x$ :

$$
\nabla \varphi(x)=-\nabla_{x} c(x, x)=0 .
$$


Hence $\varphi$ is constant and so is $\psi$ by a similar argument. Since $\varphi(x)=\psi(x)-$ $c(x, x)$, we obtain that $\psi=\varphi=A$ for some constant $A$. Condition (8.32) then becomes:

$$
q(z(x), A)=Q(A) \text { for all } x \in \Omega
$$

this implies that $z$ is constant on $\Omega$ hence so is $f(||). * \nu$; differentiating this function yields:

$$
\int_{\Omega} f^{\prime}(|x-y|) \frac{x-y}{|x-y|} \nu(y) d y=0 \text { for all } x \in \Omega .
$$

The smoothness of $f$ permits to extend the previous identity to the boundary of $\Omega$. Now let $x_{0} \in \bar{\Omega} \cap \partial \operatorname{co}(\Omega)$ and let $p \in \mathbb{R}^{2}, p \neq 0$ be such that:

$$
\left\langle p, x_{0}\right\rangle=\min \{\langle p, y\rangle: y \in \operatorname{co}(\Omega)\} .
$$

Take $x=x_{0}$ in (8.33) and take the inner product with $p$ to obtain

$$
\int_{\Omega} f^{\prime}\left(\left|x_{0}-y\right|\right)\left\langle\frac{x_{0}-y}{\left|x_{0}-y\right|}, p\right\rangle \nu(y) d y=0
$$

since the integrand is positive on $\Omega$ we get the desired contradiction.

\section{References}

[1] M. Berliant, S-K Peng, P. Wang, "Production externalities and urban configuration". JET 104 (2002). p. 275-303

[2] Y. Brenier, Polar factorization and monotone rearrangement of vectorvalued functions, Comm. Pure Appl. Math. 444 (1991), pp. 375-417.

[3] R.J. McCann, W. Gangbo, The Geometry of Optimal Transportation, Acta Math., vol. 177 (1996), pp.113-161.

[4] G. Carlier, Duality and existence for a class of mass transportation problems and economic applications, Adv. in Math. Econ., vol. 5 (2003), pp. $1-21$.

[5] K. Deimling, Nonlinear Functional Analysis, Springer-Verlag (1985).

[6] L.C. Evans, R.F. Gariepy Measure theory and fine properties of functions, Studies in Advanced Mathematics, CRC Press, Boca Raton (1992).

[7] M. Fujita, Urban economic theory, Cambridge University Press (1989) 
[8] M. Fujita, P. Krugman, A.J. Venables, The Spatial Economics, MIT Press (1999).

[9] M. Fujita and J. Thisse, Economics of Agglomeration, Cambridge University Press (2002)

[10] M. Fujita and M. Ogawa, "Multiple equilibria and structural transition of non-monocentric urban configuration", Regional Science and Urban Economics vol 12 (1982) p, 161-196

[11] W. Gangbo. An elementary proof of the polar factorization of vectorvalued functions, Arch. Rational Mech. Anal., vol. 128 (1994), pp.381399.

[12] V. Levin. Abstract cyclical monotonicity and Monge solutions for the general Monge-Kantorovich Problem, Set-Valued Analysis, vol. 7 (1999), pp.7-32.

[13] V. Levin. Reduced cost functions and their applications, Journal of Mathematical Economics, vol. 18 (1997), pp.155-186.

[14] R. E. Lucas, Jr. and E. Rossi-Hansberg, On the Internal Structure of Cities, Econometrica, vol. 70 (2002), pp. 1445-1476.

[15] M.Ogawa and M. Fujita, "Equilibrium land use patterns in a monocentric city" Journal of Regional Science vol 20 (1980) p.455-475

[16] P.A. Samuelson, "The transfer problem and transport costs: the terms of trade when impediments are absent", Economic Journal 62 (1952), pp. 278-304.

[17] S.T. Rachev, L. Rüschendorf. Mass Transportation Problems. Vol. I: Theory; Vol. II : Applications, Springer-Verlag (1998).

[18] E. Rossi-Hansberg, Optimal urban land use and zoning, to appear.

[19] J.H. von Thünen, Der isolierte Staat in Beziehung auf Landschaft und Nationalökonomie, Hamburg, 1826; English translation, by C.M. Wartenberg, von Thünen's isolated state, Oxford, Pergamon Press (1966).

[20] C. Villani, Topics in optimal transportation, Graduate Studies in Mathematics, 58, American Mathematical Society, Providence, RI, (2003). 